University of Nebraska - Lincoln

DigitalCommons@University of Nebraska - Lincoln

Roger Kirby Publications

Research Papers in Physics and Astronomy

July 1970

Far-Infrared Properties of Lattice Resonant Modes. IV.

Paraelectric Impurities

Roger D. Kirby

University of Nebraska-Lincoln, rkirby1@unl.edu

A.E. Hughes

University of Nebraska-Lincoln

A.J. Sievers

University of Nebraska-Lincoln

Follow this and additional works at: https://digitalcommons.unl.edu/physics_kirby

Part of the Physics Commons

Kirby, Roger D.; Hughes, A.E.; and Sievers, A.J., "Far-Infrared Properties of Lattice Resonant Modes. IV. Paraelectric Impurities" (1970). Roger Kirby Publications. 33.

https://digitalcommons.unl.edu/physics_kirby/33

This Article is brought to you for free and open access by the Research Papers in Physics and Astronomy at DigitalCommons@University of Nebraska - Lincoln. It has been accepted for inclusion in Roger Kirby Publications by an authorized administrator of DigitalCommons@University of Nebraska - Lincoln. 
${ }^{21}$ S. Kawano, K. Kosuge, and S. Kachi, J. Phys. Soc. Japan 21, 2744 (1966) .

${ }^{22}$ I. G. Austin, Phil. Mag. 7, 961 (1962).

${ }^{23}$ S. Westmann and C. Nordmark, Acta Chem. Scand. $14,465(1960)$.

${ }^{24}$ S. M. Ariya and M. V. Golomolzina, Fiz. Tverd. Tela 4 , 2921 (1962) [Soviet Phys. Solid State $\underline{4}, 2124$ (1963)].

${ }^{25}$ W. Klemm and L. Grimm, Z. Anorg. Allgem. Chem. 250, $42(1940)$.

${ }^{26}$ M. A. Gurevich and B. F. Ormont, Zh. Neorgan. Khim. 2, 2581 (1957).

${ }^{27}$ P. V. Geld, S. I. Alyamovskii, and I. I. Matveenko, Zh. Struet. Kheim. 2, 301 (1961).

${ }^{28}$ F. J. Morin, Phys. Rev. Letters 3, 34 (1959).

${ }^{29}$ W. W. Warren, G. A. Miranda, and W. G. Clark, Bull. Am. Phys. Soc. 12, 1117 (1967).

${ }^{30} \mathrm{~J}$. B. Goodenough (private communication).

${ }^{31} \mathrm{~J}$. Stringer, J. Less Common Metals $\underline{8}, 1$ (1965).

${ }^{32}$ J. M. Tyler, T. E. Norwood, and J. L. Fry, Phys. Rev. B 1, 297 (1970).
${ }^{33} \mathrm{E}$. Clementi, Tables of Atomic Wave Functions (IBM Corp., San Jose, 1965).

${ }^{34}$ J. C. Slater, Phys. Rev. 81, 385 (1951); see also J. C. Slater, T. M. Wilson, and J. H. Wood, ibid. 179, 28 (1969).

${ }^{35}$ Since these integrals are two-center integrals, they may be done without difficulty for the neutral atom potentials using a real space representation of the potential; in fact, exactly for our potential. This has been done for nickel, and the approximation has been proved valid. For a self-consistent calculation involving some degree of ionicity the Fourier series is needed, and methods have now been devised to accelerate convergence of the series for crystal-field integrals.

${ }^{36}$ A. B. Kunz, Phys. Rev. 180, 934 (1969).

${ }^{37} \mathrm{~J}$. Callaway and H. M. Zhang, Phys. Rev. B 1 , 305 (1970).

${ }^{38} \mathrm{~J}$. Yamashita, S. Asano, and S. Wakoh, J. Appl。 Phys. 39, 1274 (1968); see also Ref. 2 .

${ }^{39} \mathrm{~A}$ similar result was obtained for paramagnetic NiO. See Ref. 14.

\title{
Far-Infrared Properties of Lattice Resonant Modes. IV. Paraelectric Impurities*
}

\author{
R. D. Kirby, ${ }^{\dagger}$ A. E. Hughes, ${ }^{+}$and A. J. Sievers \\ Laboratory of Atomic and Solid-State Physics, Cornell University, Ithaca, New York 14850 \\ (Received 27 February 1970)
}

\begin{abstract}
We have studied the three paraelectric defect systems $\mathrm{KCl}: \mathrm{Li}^{+}, \mathrm{RbCl}: \mathrm{Ag}^{+}$, and $\mathrm{NaCl}: \mathrm{OH}^{-}$ through the temperature dependence and electric field dependence of their far-infrared absorption spectra. It is found that the motional energy states for the defects in these three systems have many properties in common. In each case the impurity ion has an associated electric dipole moment and low-lying tunneling energy levels. The defects also have higher energy levels which can be attributed, at least in part, to c. m. resonant-mode motion. The $\mathrm{KCl}: \mathrm{Li}^{+}$ tunneling levels are found to be well described by the model of Gomez, Bowen, and Krumhansl, assuming a $\mathrm{Li}^{+}$dipole moment of $1.14 \pm 0.05 e \AA$ for both $\mathrm{Li}^{6}$ and $\mathrm{Li}^{7}$. The most surprising feature of the $\mathrm{KCl}$ : $\mathrm{Li}^{+}$higher-lying energy levels is that they show a negative isotope shift [i.e., $\bar{\omega}\left(\mathrm{Li}^{6}\right)<\bar{\omega}\left(\mathrm{Li}^{7}\right)$ ]. The three observed absorption lines in $\mathrm{RbCl}$ : $\mathrm{Ag}^{+}$, as well as their dependence on an external electric field, are shown to be consistent with a [110] off-center position for the $\mathrm{Ag}^{+}$ions. The far-infrared absorption spectrum of $\mathrm{NaCl}: \mathrm{OH}^{-}$is complicated, with at least eight absorption lines between 2 and $22 \mathrm{~cm}^{-1}$. Considerable agreement is found between the experimental results and a model which incorporates both librational tunneling of the $\mathrm{OH}^{-}$ion and c.m. vibrational motion.
\end{abstract}

\section{INTRODUCTION}

The first three papers of this series ${ }^{1-3}$ have been concerned with various properties of lattice resonant modes of monatomic impurities in alkali halides. Each defect-lattice system studied in these earlier works has been confirmed to be of the on-center type, where the impurity ion substitutes for a host ion at the normal lattice site. In this paper, we wish to discuss the properties of the far-infrared absorption spectra due to para- electric impurities in alkali halides, with particular emphasis on the motional energy states which can arise from c. m. resonant-mode motion. As will be seen, the far-infrared properties of paraelectric defect systems can be strikingly different than those of on-center impurities.

Paraelectric defects may be divided into two classes. Historically, the first class consists of dipolar diatomic impurities such as $\mathrm{OH}^{-}$and $\mathrm{CN}^{-}$. In these cases, the impurity molecule has several equivalent preferred directions of dipolar align- 
ment in a particular symmetry direction of the host crystal. It has been shown ${ }^{4,5}$ that quantummechanical tunneling of the molecule between the equilibrium orientations results in closely spaced tunneling energy levels. Because of their dipolar nature, the impurity ions tend to be aligned by external electric fields. This leads to a Stark splitting of the tunneling levels.

The second, and in principle less complicated, class of paraelectric defects consists of monovalent monatomic impurities which enter the host lattice substitutionally, but do not occupy the normal host-ion lattice sites. Instead, the impurity ion has several equivalent equilibrium sites (positions of minimum potential energy), displaced from the host-ion cavity center along a symmetry direction of the crystal. Undoubtedly, the most thoroughly studied system of this class has been $\mathrm{KCl}: \mathrm{Li}^{+}$, in which the $\mathrm{Li}^{+}$impurity ion has eight equivalent equilibrium sites off center in the [111] crystallographic directions. Gomez, Bowen, and Krumhansl ${ }^{6}$ have shown that tunneling of the impurity ion between these sites leads to low-lying tunneling energy levels. Classically, the $\mathrm{Li}^{+}$ion has a permanent electric dipole moment, formed by the separation of the positive $\mathrm{Li}^{+}$ion from the effectively negative $\mathrm{K}^{+}$vacancy center. As for paraelectric systems of the first class, this dipole moment leads to a strong dependence of the tunneling levels on external electric fields.

While the tunneling properties of paraelectric impurities can be studied with a variety of experimental techniques, including dielectric-constant measurements, ${ }^{7,8}$ paraelectric cooling, ${ }^{9-12}$ paraelectric resonance, ${ }^{13-16}$ nuclear magnetic resonance, ${ }^{17}$ thermal-conductivity measurements, ${ }^{18-21}$ ultrasonic-attenuation measurements, ${ }^{22}$ and specific-heat measurements, ${ }^{23}$ the possible higherenergy states are not so accessible for investigation. For this reason, there is very little data available on these states. Sievers ${ }^{24}$ found an absorption band near $40 \mathrm{~cm}^{-1}$ in $\mathrm{KCl}: \mathrm{Li}^{+}$, and $\mathrm{Nolt}^{25}$ observed three absorption lines between 20 - and 40- $\mathrm{cm}^{-1} \mathrm{RbCl}: \mathrm{Ag}^{+}$. $\mathrm{Renk}^{26}$ observed $\mathrm{OH}^{-}$-induced absorption lines in the KI phonon gap, Bosomworth ${ }^{27}$ measured the far-infrared absorption spectra due to $\mathrm{OH}^{-}$and $\mathrm{OD}^{-}$in several alkali halides, and we have reported some preliminary results $^{1,28}$ for $\mathrm{NaCl}: \mathrm{OH}^{-}$. For molecular impurities the experimental difficulties are at least partially alleviated because motional energy states lying below $100 \mathrm{~cm}^{-1}$ can often be studied as sidebands of the molecular-vibrational stretching modes, which typically fall between 1000 and $4000 \mathrm{~cm}^{-1}$. The most complete investigations of this type include the study of $\mathrm{CN}^{-}$in alkali halides by Seward and Narayanamurti, ${ }^{21}$ and the study of $\mathrm{OH}^{-}$in alkali halides by Wedding and Klein. ${ }^{29,30}$

The far-infrared absorption spectrum arising from a paraelectric impurity in an alkali halide is expected to be more complicated than that due to an on-center monatomic impurity. In order to interpret such spectra, it is necessary to know what types of motional energy states are possible in the spectral region of interest. Because of the absence of rotational degrees of freedom, the case of an off-center monatomic impurity is in principle less complicated than that of a diatomic impurity. Here, we will give a brief qualitative description of the possible motional states which are common to both classes of paraelectric impurities. The additional complications which must be considered for diatomic impurities will be discussed later in this paper.

Since the simple case of the three-dimensional harmonic oscillator explains many features of the resonant-mode absorption by on-center impurities, we will use it as a starting point for our description of paraelectric impurities. In this picture, the ground-state motion of the defect oscillator in the cubic crystal field of the host lattice transforms as the $A_{1 g}$ irreducible representation of the $O_{h}$ symmetry group, and the first excited state transforms as the $T_{1 u}$ representation. The transition between these two energy levels is electric dipole allowed, and is assumed to give rise to the observed resonant-mode absorption line.

We now consider what happens if this oscillator, instead of being an on-center monatomic defect in the cubic lattice, is a paraelectric impurity. It is then characterized by its dipole orientation, that is, the off-center displacement direction for a monatomic impurity, or the direction of the molecular axis orientation for a diatomic impurity. In either case, the c.m. vibrational motion of the impurity occurs in a local potential well of lower symmetry than the octahedral symmetry of the lattice. In terms of the simple dipole orientation directions [100], [111], and [110], the local symmetries are $C_{4 v}, C_{3 v}$, and $C_{2 v}$, respectively. Under these conditions, the oscillator cannot be characterized as spherical, so that the resonant-mode excited state is no longer triply degenerate. For all three local symmetries, the resonant-mode ground state transforms as the $A_{1}$ irreducible representation of the appropriate symmetry group. For both $C_{3 v}$ and $C_{4 v}$, the resonant-mode excited state consists of two levels, a singlet $A_{1}$ and a doublet $E$. For $C_{2 v}$, there are three levels transforming as $A_{1}, B_{1}$, and $B_{2}$. Physically, it is clear that this kind of analysis is valid only if the potential-energy barriers hindering the dipole orientation from moving between the equivalent equilibrium sites are larger than the energies of 
the resonant-mode excited states.

The next step in this analysis is to take into account tunneling of the impurity ion between the equilibrium sites. This has been done for the ground state by several authors. ${ }^{4-6}$ If the smalltunneling-rate (high-barrier) approximation is also assumed to be valid for the resonant-mode excited-state levels, then the tunneling properties of these levels are easily calculated. Admittedly such a calculation cannot be used in every case, but we have found it to be useful in interpreting the properties of the far-infrared absorption spectra of certain paraelectric impurities. This is the approach that we will use in our analysis of the far-infrared properties of $\mathrm{KCl}: \mathrm{Li}^{+}, \mathrm{RbCl}: \mathrm{Ag}^{+}$, and $\mathrm{NaCl}: \mathrm{OH}^{-}$.

In Sec. II, the experimental techniques and apparatus used in this investigation are described, and in Sec. III, the experimental results on $\mathrm{KCl}: \mathrm{Li}^{+}$are compared with the model of Gomez et al. In Sec. IV, some preliminary experimental results on $\mathrm{RbCl}: \mathrm{Ag}^{+}$are presented and compared with the expected properties of a [110] defect. Section $\mathrm{V}$ contains the experimental results on $\mathrm{NaCl}: \mathrm{OH}^{-}$and the interpretation of these results in terms of a model which includes both resonantmode energy states and librational-tunneling energy states.

\section{EXPERIMENT}

Paraelectric-defect systems exhibit some unique features which make their study in the far-infrared spectral region especially interesting. However, to effectively study these systems, it has been necessary to make use of some of the more recent advances in far-infrared spectroscopy, including a lamellar Fourier interferometer ${ }^{31}$ and a liquid $\mathrm{He}^{3}$-cooled germanium bolometer. ${ }^{32}$

As was pointed out in Sec. I, paraelectric-defect systems can have many motional states in the far infrared, arising from a combination of resonant-mode motion and tunneling motion. The types of experiments most likely to give useful information about these energy states are obvious. The presence of closely spaced tunneling levels will cause the strengths of the many (resonant-mode + tunneling) transitions to vary rapidly when the temperature is comparable to the ground-state tunneling splittings. Similarly, the electric dipole moment associated with the defect should cause a strong dependence on external electric fields.

The doped single-crystal alkali-halide samples were grown in an argon atmosphere using the Kyropolous technique. ${ }^{33}$ After being pulled from the melt, the crystals were cooled slowly in about $4 \mathrm{~h}$ to room temperature, after which they were uti- lized without further heat treatment.

The impurity-induced far-infrared absorption spectra were obtained by comparing the transmission through a doped sample with that through the pure host crystal. Using a $\mathrm{He}^{3}$-cooled bolometer, it was possible to take data down to $2 \mathrm{~cm}^{-1}$.

For temperature-dependence measurements, the samples to be studied were mounted in an evacuable chamber, coupled to the $1.2^{\circ} \mathrm{K}$ outer helium bath by $\mathrm{He}$ exchange gas. For tempera tures above $1.2^{\circ} \mathrm{K}$, the samples were heated by passing current through a heater on the sample mount, and the temperature was measured by monitoring the resistance of a $1-\mathrm{k} \Omega \frac{1}{10}-\mathrm{W}$ AllenBradley resistor which had been mounted in good thermal contact with the sample.

As will be seen, the far-infrared absorption spectrum of $\mathrm{NaCl}: \mathrm{OH}^{-}$has been measured at a temperature of $0.6{ }^{\circ} \mathrm{K}$. For these measurements, a single $\mathrm{He}^{3}$ cryostat was used to cool both the pure and doped single-crystal samples, as well as to cool the germanium bolometer. The lowest sample temperatures were obtained with samples which had been pressed against an indium pad on the copper $\mathrm{He}^{3}$ can with a phosphor-bronze spring. The detector was mounted in a cylindrical cavity beneath the samples. A schematic diagram of this cryostat is shown in Fig. 1.

Since the samples, when mounted in this config-

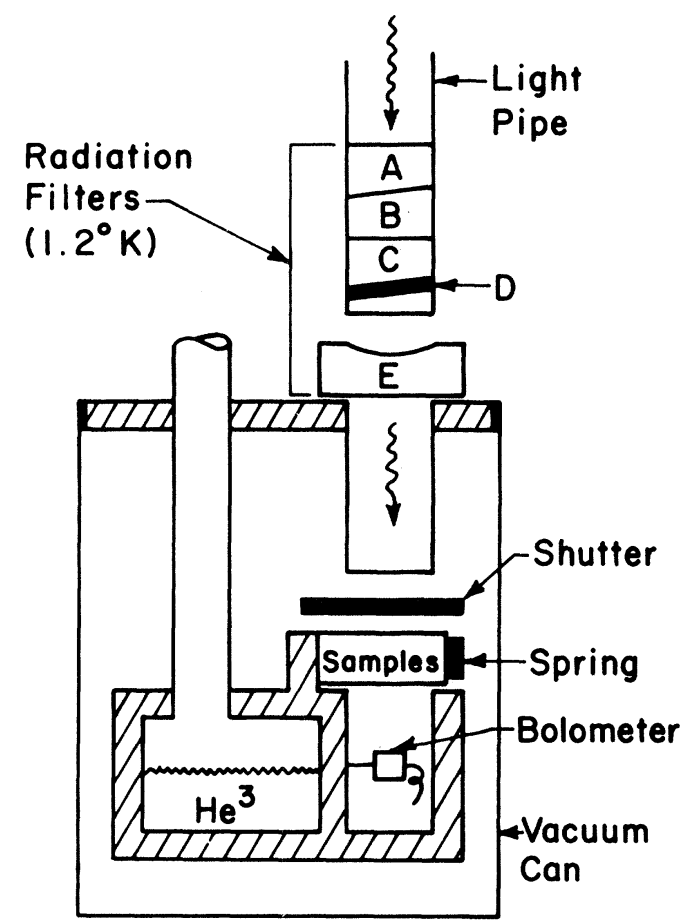

FIG. 1. Helium-3 cryostat used for cooling alkalihalide samples to $0.6^{\circ} \mathrm{K}$. The filters $\mathrm{A}, \mathrm{B}, \mathrm{C}, \mathrm{D}$, and $\mathrm{E}$ are described in the text. 
uration, are not movable, transmission measurements are first made on the pure sample. A shutter which initially covers the doped sample is then activated so as to cover the pure sample, thus allowing the doped-sample transmission spectrum to be measured.

The difficulty in making far-infrared transmission measurements at $\mathrm{He}^{3}$ temperatures lies in filtering out the $300^{\circ} \mathrm{K}$ blackbody radiation from the top of the cryostat, without severely reducing the amount of far-infrared radiation reaching the samples. For this purpose, there is a complex radiation filter immersed in $1.2^{\circ} \mathrm{K}$ helium above the sample chamber. In Fig. 1, filter A is KI, B is $\mathrm{LiF}, \mathrm{C}$ is $\mathrm{NaCl}, \mathrm{D}$ is black polyethylene, and $\mathrm{E}$ is crystal quartz. Even with this filter, the doped sample runs nearly $0.1^{\circ} \mathrm{K}$ warmer (at $0.6^{\circ} \mathrm{K}$ ) when it is in the beam than when it is covered by the shutter.

The proximity of the pure and doped samples in this cryostat allows a small leakage of light through the sample covered by the shutter. This effect tends to reduce the measured absorption coefficient. It can be corrected for by measuring the absorption of a single sample in this cryostat at both 0.6 and $1.2{ }^{\circ} \mathrm{K}$. This technique gives a "temperature-induced absorption coefficient" for the 1.2 to $0.6^{\circ} \mathrm{K}$ temperature change, and allows the leakage to be corrected for. This method, however, requires the use of a $1.2^{\circ} \mathrm{K}$ germanium bolometer mounted in thermal contact with the $\left(1.2^{\circ} \mathrm{K}\right)$ vacuum can, as opposed to a bolometer operating at $0.5^{\circ} \mathrm{K}$, resulting in less sensitivity.

The far-infrared absorption measurements with the doped sample in an external electric field were made using a special sample holder and sample geometry described by Clayman. ${ }^{34}$ For a [100] applied electric field, absorption measurements were made with the electric field vector of the incident radiation polarized either parallel or perpendicular to the dc field. For a [110] applied field, there are three distinct polarizations for the incident radiation, corresponding to $E_{\mathrm{IR}} \|\lfloor 110]$, $E_{\mathrm{IR}} \|[110]$, and $E_{\mathrm{IR}} \|[100]$. The polarizer used in all cases was a 500-line/in. Ni grid on a Mylar substrate.

Electric field measurements were made with the sample immersed in the liquid-helium bath. It was found that $30-50 \%$ higher fields could be reached with the bath at $4.2^{\circ} \mathrm{K}$ than with it at $1.2^{\circ}$ $\mathrm{K}$. Chester ${ }^{35}$ has suggested that this effect is due to the much greater mobility of electron "bubbles" and charged impurities in $\lambda$ helium. The $\mathrm{He}^{3}$ detector cryostat was designed to be used with the outer helium bath at $1.2^{\circ} \mathrm{K}$, so field-dependent measurements were made at this temperature until electrical breakdown occurred (typically $80-100 \mathrm{kV}$ / $\mathrm{cm})$. The outer bath was then refilled, and measurements were made at $4.2^{\circ} \mathrm{K}$. This resulted in higher fields (typically $110-150 \mathrm{kV} / \mathrm{cm}$ ), but at the expense of about a factor of 2 in the $\mathrm{He}^{3}$-cooled detector sensitivity due to the greater heat leak from the outer bath.

$$
\text { III. } \mathrm{KCl}: \mathrm{Li}^{+}
$$

\section{A. Experimental Results}

\section{Ground-State Tunneling Multiplet}

The experimental techniques described in Sec. II have been used to study the energy levels in the $\mathrm{KCl}: \mathrm{Li}^{+}$tunneling multiplet as a function of external electric field. It seems well confirmed at this point that the multiplet consists of four approximately equally spaced energy levels, with the separation of adjacent levels being $0.8 \mathrm{~cm}^{-1}$ for isotopic $\mathrm{Li}^{7}$ impurities ${ }^{18,36}$ and $1.15 \mathrm{~cm}^{-1}$ for $\mathrm{Li}^{6}$ impurities. ${ }^{23}$ As we shall see later in this section, the only optically active transitions are between adjacent levels of the multiplet, so that the allowed transition energies in zero field are too small to be studied with the lamellar interferometer. However, with an external electric field applied in the [100] direction and the electric field vector of the incident radiation polarized parallel to the applied field, one absorption line was obr served. It is shown for several values of the external field in Fig. 2. The sample used for these measurements was doped with $4.6 \times 10^{17} \mathrm{Li}^{6} / \mathrm{cm}^{3}$. The sample temperature was $1.2^{\circ} \mathrm{K}$ and the instrumental resolution $0.18 \mathrm{~cm}^{-1}$. We should point out here that for an interferometer, the instrumental resolution $\left(\Gamma_{\text {inst }}\right)$ cannot simply be subtracted from the observed linewidth $\left(\Gamma_{\text {obs }}\right)$ to obtain the real linewidth $\left(\Gamma_{\text {real }}\right)$. Nolt has shown empirically that it is more nearly true that

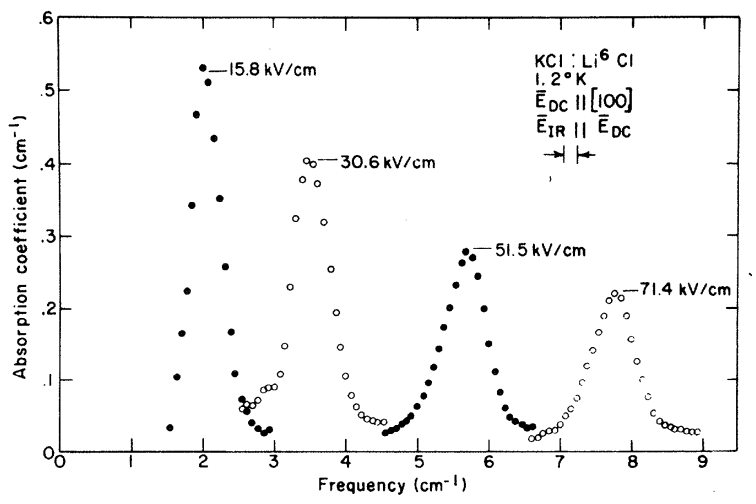

FIG. 2. $\mathrm{KCl}: \mathrm{Li}^{6} \mathrm{Cl}$ tunneling absorption line for several values of the external [100] electric field. 


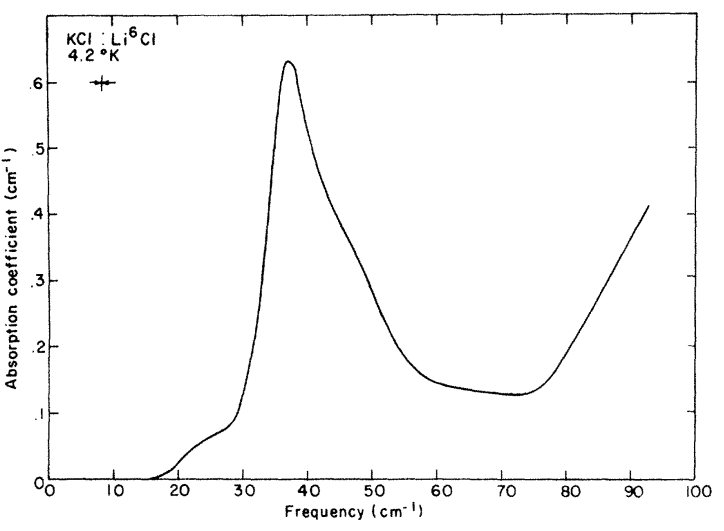

FIG. 3. $\mathrm{KCl}: \mathrm{Li}^{6} \mathrm{Cl}$ absorption spectrum.

$$
\Gamma_{\text {obs }}^{2}=\Gamma_{\text {real }}^{2}+\Gamma_{\text {inst }}^{2} .
$$

So if $\Gamma_{\text {inst }} \leq \frac{1}{3} \Gamma_{\text {real }}$, then it is a good approximation that $\Gamma_{\text {obs }}=\Gamma_{\text {real }}$. This approximation is used throughout the paper.

In Fig. 2, the absorption linewidth (full width at half-maximum absorption) is $0.7 \mathrm{~cm}^{-1}$ and has a weak dependence on the field. There is no observable fine structure even though the resolution is nearly a factor of 4 less than the linewidth. It should be noted that the absorption strength (the area under the absorption line) of the line decreases with increasing field.

These data were obtained by dividing the fieldon transmission spectrum by the zero-field transmission spectrum. For the lowest field value shown in Fig. 2 there is some overlap between the shifted absorption line and the unperturbed line. This tends to change the shape, strength, and position of the observed absorption line, but because of the narrow linewidth for this particular sample, these effects are small.

Transmission measurements were also made on $\mathrm{KCl}: \mathrm{Li}^{+}$with the $E$ vector of the incident radiation polarized perpendicular to the external [100] electric field. In this case a weak absorption line was observed to shift up in frequency with approximately the same slope as the line observed in parallel polarization. However, the strength of this line was only about $5 \%$ of that of the line in parallel polarization, and it also decreased in strength with increasing field. These two facts suggest that the absorption in perpendicular polarization arises from a small leakage of radiation polarized parallel to the applied field, or from a small component of the applied field in the perpendicular polarization direction.

With the external electric field applied in the [110] crystallographic direction, again only one absorption line was observed. Its field dependence was qualitatively the same as that for a [100] field. For the polarization directions $E_{\mathrm{IR}} \| E_{\mathrm{dc}}$ and $E_{\mathrm{IR}} \|$ [110], the field dependences of the absorption frequency and strength were identical. The third distinct polarization direction ( $E_{\mathrm{IR}} \|$ [100]) was not studied.

\section{Resonant-Mode Excited States}

a. Temperature dependence. Figure 3 shows the absorption spectrum of $\mathrm{KCl}: \mathrm{Li}^{6} \mathrm{Cl}$ between 5 and $90 \mathrm{~cm}^{-1}$. The spectrum consists of an asymmetric peak near $40 \mathrm{~cm}^{-1}$, with a full width at halfmaximum absorption of about $15 \mathrm{~cm}^{-1}$. The increase in absorption above $75 \mathrm{~cm}^{-1}$ is attributed to one-phonon absorption and will not be considered further. No sharp structure is seen in the absorption spectrum for a resolution of $0.2 \mathrm{~cm}^{-1}$. This is somewhat surprising since it is known that the ground-state tunneling multiplet contains energy levels separated by $1.15 \mathrm{~cm}^{-1}$, and transitions between it and the excited states should reflect this structure.

Figure 4 shows the $\mathrm{KCl}: \mathrm{Li}^{6} \mathrm{Cl}$ absorption spectrum for temperatures of 1.15 and $4.2^{\circ} \mathrm{K}$. On this expanded scale, and especially at the lower temperature, the absorption band appears to consist of two peaks, one near $38 \mathrm{~cm}^{-1}$ and the other near $47 \mathrm{~cm}^{-1}$. The separation of these two peaks is too great to be due to the structure in the ground-state tunneling multiplet, and must be attributed to the energy-level structure of the excited state. The oscillator strength of this band at $4.2^{\circ} \mathrm{K}$ is measured to be $f=0.043$. By comparison, the oscillator strength of the $\mathrm{KBr}: \mathrm{Li}^{+}$resonant mode is 0.1 .

We note that the absorption peak at $38 \mathrm{~cm}^{-1} \mathrm{de}-$ creases in strength with decreasing temperature, while the strength of the higher-frequency peak remains approximately constant. These effects are reflected in the position of the centroid of ab-

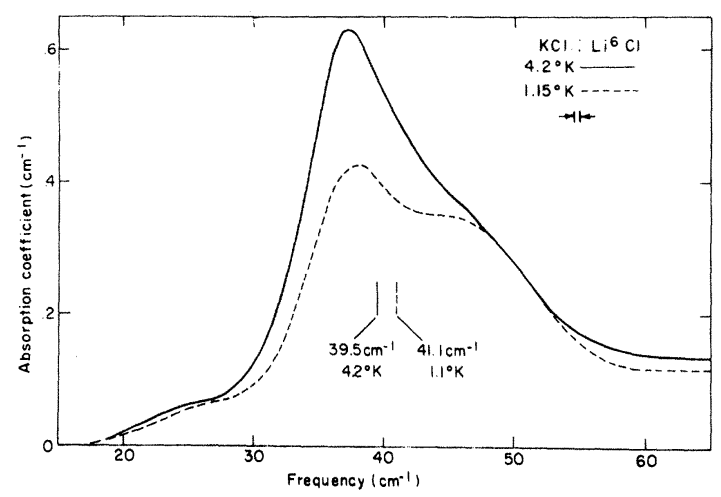

FIG. 4. Temperature dependence of the $\mathrm{KCl}: \mathrm{Li}^{6} \mathrm{Cl}$ $40-\mathrm{cm}^{-1}$ absorption band. This sample contained 2.4 $\times 10^{18} \mathrm{Li}^{6}$ ions $/ \mathrm{cm}^{3}$. 


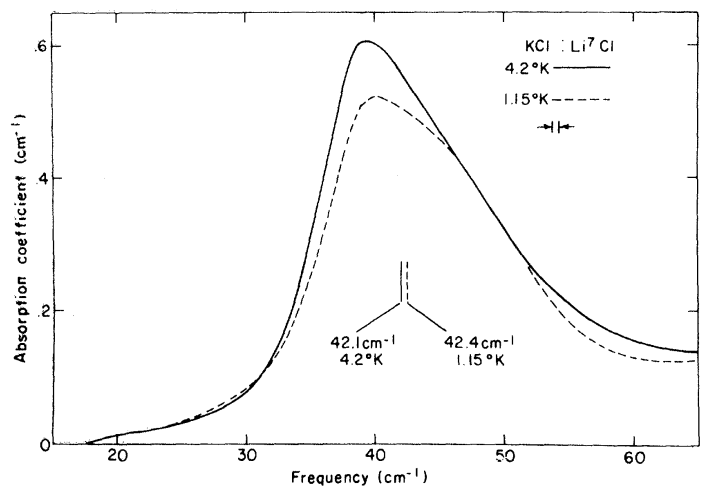

FIG. 5. Temperature dependence of the $\mathrm{KCl}: \mathrm{Li}^{7} \mathrm{Cl}$ $40-\mathrm{cm}^{-1}$ absorption band. This sample contained 2.3 $\times 10^{18} \mathrm{Li}^{7}$ ions $/ \mathrm{cm}^{3}$.

sorption, which as shown in the figure, moves up in frequency with decreasing temperature. Such a rapid temperature dependence of the absorption spectrum is to be expected because the relative Boltzmann populations of the levels in the tunneling multiplet are changing rapidly in this temperature region.

In Fig. 5 is shown the temperature dependence of $\mathrm{Li}^{7}$-doped $\mathrm{KCl}$. Again, the absorption band is not symmetric, but here two separate peaks in absorption are not so clearly defined. However, the absorption near $39 \mathrm{~cm}^{-1}$ does decrease in strength with decreasing temperature, and near $47 \mathrm{~cm}^{-1}$ the absorption is approximately constant for these two temperatures. The centroid of absorption for $\mathrm{Li}^{7}$ impurities is also at a higher frequency for the lower temperature.

The increase in absorption upon going from 1.15 to $4.2^{\circ} \mathrm{K}$ is shown in Fig. 6 for both $\mathrm{Li}^{6}$ and $\mathrm{Li}^{7}$ impurities. In both cases, this "temperature-induced absorption" appears to be symmetric with a full width at half-maximum absorption of 8.4 $\mathrm{cm}^{-1}$. Such a large width may indicate that several transitions are contributing to the absorption. These temperature-induced changes in absorption must be due to changes in the relative Boltzmann populations of the ground-state tunneling levels.

b. Isotope shift. As can be seen from Figs. 4 and 5 , the $\mathrm{Li}^{7}$ absorption centroid lies higher in frequency than the $\mathrm{Li}^{6}$ centroid for both 1.15 and $4.2{ }^{\circ} \mathrm{K}$. This negative isotope shift is unexpected, since for the four (on-center) lattice resonantmode systems which have been the subject of the most study ${ }^{1,37}$ a positive isotope shift is observed, that is, the lighter isotope has the higher resonant frequency. The shift in each case is equal to or greater than that predicted for an Einstein oscillator; namely,

$$
\omega_{2} / \omega_{1}=\left(m_{1} / m_{2}\right)^{1 / 2},
$$

where $m_{1}$ and $m_{2}$ are the isotopic impurity masses, and $\omega_{1}$ and $\omega_{2}$ are the corresponding resonant frequencies.

This type of calculation is not very meaningful for an off-center defect because of the presence of tunneling levels in both the ground state and (presumably) the excited state, which makes a single resonant frequency difficult to define. In this case, Eq. (1) can only give an indication of the isotope shift for the energy centroids of the ground- and excited-state tunneling multiplets. By itself, it tells us very little about the isotope shifts of the (many) individual transitions between the ground- and excited-state multiplets. We know that the tunneling levels for the $\mathrm{KCl}: \mathrm{Li}^{+}$ ground state exhibit a large positive isotope shift $(\sim 40 \%),{ }^{23}$ so it would not be unexpected if some of the ground-excited transitions had an effective negative isotope shift. (In fact, Fig. 6 may show an example of this effect.) As we shall see later in this section, the difficulty in explaining the $\mathrm{KCl}: \mathrm{Li}^{+}$isotope shift in detail arises because the $40-\mathrm{cm}^{-1}$-band absorption centroid shows a negative isotope shift.

Besides $\mathrm{KCl}: \mathrm{Li}^{+}$, only one other resonantmode system has been found which shows an isotope shift which deviates markedly from that calculated from Eq. (1). Sievers ${ }^{24}$ and Macdonald et $a l .{ }^{38}$ have observed the resonant-mode absorption line at $43.7 \mathrm{~cm}^{-1}$ in $\mathrm{NaCl}: \mathrm{Li}^{7} \mathrm{Cl}$. For $\mathrm{NaCl}: \mathrm{Li}^{6}$ $\mathrm{Cl}$, we have measured an absorption frequency of $45.3 \mathrm{~cm}^{-1}$, corresponding to an isotope shift of $1.6 \mathrm{~cm}^{-1}$. Equation (1) predicts an isotope shift of $3.5 \mathrm{~cm}^{-1}$. The absorption lines for both lithium isotopes are shown in Fig. 7. The $\mathrm{Li}^{+}$concentrations for these two samples are $6.0 \times 10^{19}$ and 5.9

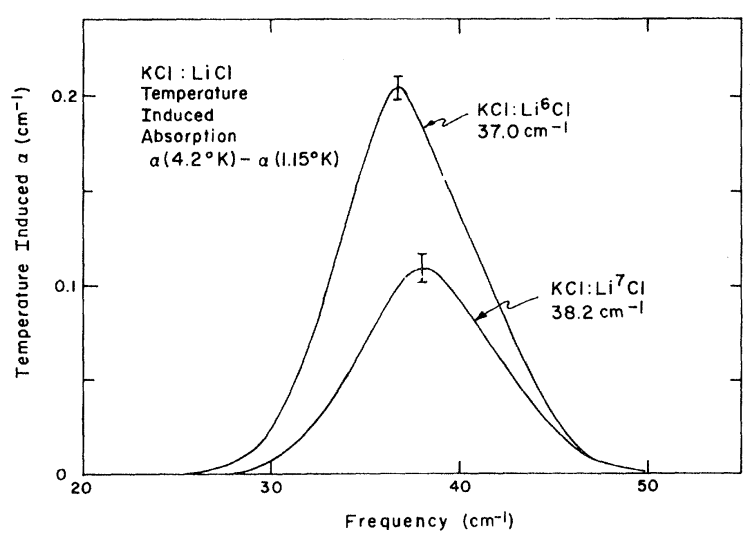

FIG. 6. 1.15 to $4.2^{\circ} \mathrm{K}$ temperature-induced absorption spectra for $\mathrm{KCl}$ : $\mathrm{Li}^{6} \mathrm{Cl}$ and $\mathrm{KCl}: \mathrm{Li}^{7} \mathrm{Cl}$. These data were obtained by subtracting the $1.15^{\circ} \mathrm{K}$ absorption spectrum from the $4.2^{\circ} \mathrm{K}$ absorption spectrum. 


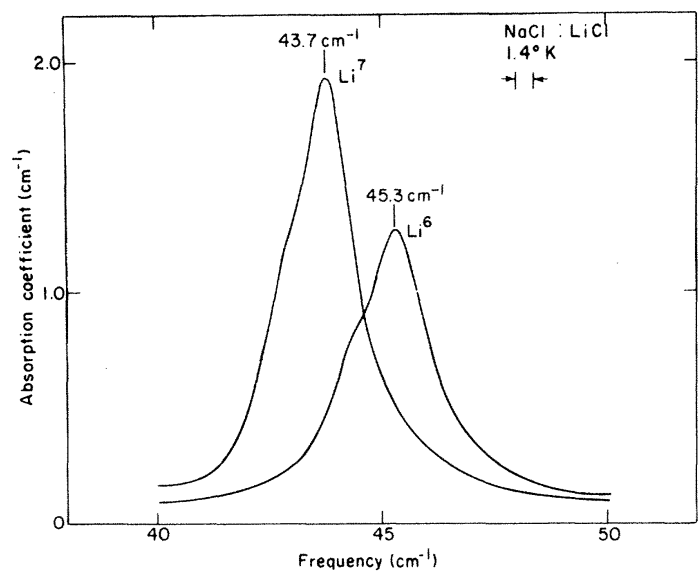

FIG. 7. NaCl: $\mathrm{Li}^{+}$resonant-mode isotope shift.

$\times 10^{19} \mathrm{Li}^{7} / \mathrm{cm}^{3}$, respectively. From these concentrations and from the areas under the absorption lines, we calculate an oscillator strength of $\sim 2.7$ $\times 10^{-4}$, which is indeed very small. (For $\mathrm{KBr}: \mathrm{Li}^{+}$ and $\mathrm{NaCl}: \mathrm{Cu}^{+}$the oscillator strengths are 0.1 and 0.4, respectively.) Since the dependence of the absorption strength on impurity concentration has not been measured, some caution must be exercised in ascribing this line to a single-defect resonant-mode transition.

It should be noted that for neither isotope is the absorption line symmetric. The small bump on the low-frequency side of each absorption line could indicate that $\mathrm{NaCl}$ : $\mathrm{LiCl}$ is also an off-center system, and that the relatively low resolution for the data in Fig. 7 was not sufficient to resolve the tunneling structure. However, the temperature dependence between 1.2 and $4.2^{\circ} \mathrm{K}$ of this absorption shows only a very slight uniform change in strength, as would be expected for an on-center defect. Although we suspect that the defect is on center and the asymmetry stems from the two $\mathrm{Cl}^{-}$ isotopes in the host lattice, it is interesting to note that point-ion-potential calculations by Wilson et $a l .{ }^{39}$ indicate that $\mathrm{Li}^{+}$should be off center in $\mathrm{NaCl}$.

The electric field dependence of the $\mathrm{KCl}: \mathrm{Li}^{+}$ excited-state absorption band has also been measured. As these results are not conclusive enough to play an important role in the understanding of this system, they are presented in the Appendix.

\section{B. Model}

The intention here is to summarize some of the main features of the model developed by Gomez, Bowen, and Krumhansl (hereafter referred to as GBK) to describe the tunneling motion of an offcenter impurity. We will also include the extension of the model to the $\mathrm{KCl}: \mathrm{Li}^{+}$resonant-mode excited state in the manner indicated in Sec. I.

To be concise, we will consider here only impurities displaced from the host-ion cavity center in the [111] crystallographic directions, since this is the particular case that appears to be appropriate for $\mathrm{KCl}: \mathrm{Li}^{+}$. We first consider Fig. 8, which shows a schematic representation of the eight equivalent [111] equilibrium positions for the displaced impurity ion. We initially want to describe the motion of the impurity when it is localized in one of these sites, and then look for approximate solutions to the motion in the multiwell potential in terms of linear combinations of the single-well "basis-state" eigenfunctions. To do this, we must solve for the motion of the impurity ion in a potential well which closely approximates the local potential wells at the equilibrium sites, but which effectively prevents the impurity from leaving the immediate vicinity of the equilibrium position. GBK assumed harmonic local potentials in order to do numerical calculations, but since we are concerned here with the qualitative features of the tunneling approximation, no such assumption is required. In fact, the only requirement necessary is that the tunneling rate between the equilibrium sites be small compared to the "resonant frequency" of the impurity ion in the local wells.

With the preceding discussion in mind, we take $V_{a}(x, y, z)$ to be the approximate potential-energy distribution for the impurity when in site $a$. The Hamiltonian for the impurity when in site $a$ is then

$$
H_{a}=P_{a}^{2} / 2 m+V_{a}(x, y, z),
$$

where $m$ is the impurity mass. There are similar equations for $H_{b}, \ldots, H_{h}$.

There are restrictions on $H_{a}, \ldots, H_{h}$ because of the symmetry of the multiwell potential. First, $H_{a}, \ldots, H_{h}$ must exhibit the $C_{3 v}$ local symmetry of the equilibrium sites. Second, because of the cubic symmetry of the alkali-halide lattice, the Hamiltonians $H_{a}, \ldots, H_{h}$ must be related by the

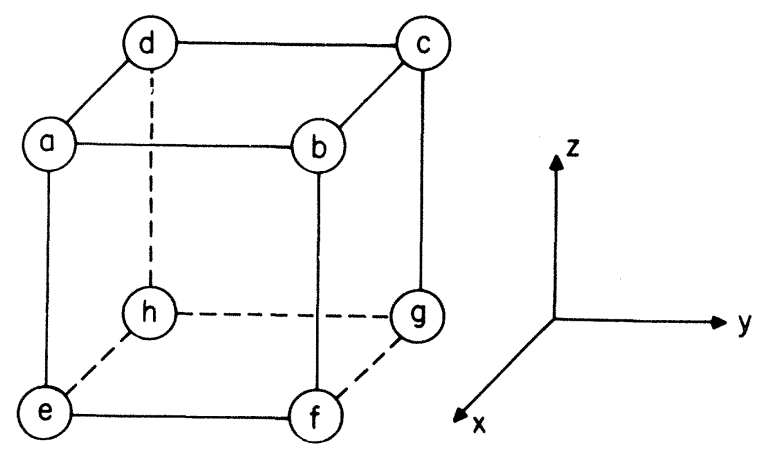

FIG. 8. Schematic representation of the $\mathrm{Li}^{+}$off-center equilibrium sites in $\mathrm{KCl}$. 
symmetry operations of the octahedral group $O_{h}$. That is, for example, $H_{b}$ can be obtained from $H_{a}$ by rotating $H_{a}$ through $90^{\circ}$, so that

$$
H_{b}(y,-x, z)=H_{a}(x, y, z) \text {. }
$$

If the ground-state solution to Eq. (2) is

$$
H_{a}|a\rangle=E_{0}|a\rangle,
$$

then the ground-state eigenvector $|b\rangle$ for $H_{b}$ must be related to $|a\rangle$ by a rotation through $90^{\circ}$, and the corresponding eigenvalue must be $E_{0}$. Thus, the ground state for this eight-well system in the absence of tunneling is eightfold degenerate with energy $E_{0}$ and eigenvectors $|a\rangle,|b\rangle, \ldots,|h\rangle$.

The resonant-mode excited state for the impurity in one of the local wells consists of two levels, corresponding to the $A_{1}$ and $E$ irreducible representations of the $C_{3 v}$ symmetry group. We denote the eigenvectors for the eight $A_{1}$-symmetry excited-state levels by $\left|a^{\prime}\right\rangle,\left|b^{\prime}\right\rangle, \ldots\left|h^{\prime}\right\rangle$, and the doubly degenerate eigenvectors for the eight $E$ symmetry excited-state levels by $\left|a^{\prime \prime}\right\rangle$ and $\left|a^{\prime \prime \prime}\right\rangle$, $\left|b^{\prime \prime}\right\rangle$ and $\left|b^{\prime \prime \prime}\right\rangle, \ldots,\left|h^{\prime \prime}\right\rangle$ and $\left|h^{\prime \prime \prime}\right\rangle$. These eigenvectors are also solutions to the corresponding approximate Hamiltonians $H_{a}, H_{b}, \ldots, H_{h}$, so that, for example,

$$
\begin{aligned}
& H_{a}\left|a^{\prime}\right\rangle=E_{A}\left|a^{\prime}\right\rangle, \\
& H_{a}\left|a^{\prime \prime}\right\rangle=E_{E}\left|a^{\prime \prime}\right\rangle, \\
& H_{a}\left|a^{\prime \prime \prime}\right\rangle=E_{E}\left|a^{\prime \prime \prime}\right\rangle .
\end{aligned}
$$

The effect of allowing the impurity ion to tunnel between the equilibrium sites is included by noting that there will be matrix elements of $H$, the real multiwell Hamiltonian, connecting the basis-state eigenvectors. In the approximation that the matrix elements between the ground-state basis states and the excited-state basis states can be neglected, we may treat the ground-state tunneling problem by itself. Then for the eightfold-degenerate ground state there are only three possible different types of Hamiltonian matrix elements for this system. They are of the forms

$$
\begin{aligned}
\langle a|H| b\rangle & =\langle b|H| c\rangle \\
& =\cdots \text { (edge tunneling), } \\
\langle a|H| c\rangle & =\langle b|H| d\rangle \\
& =\ldots \text { (face-diagonal tunneling), } \\
\langle a|H| g\rangle & =\langle b|H| h\rangle \\
& =\ldots \text { (body-diagonal tunneling). }
\end{aligned}
$$

The configuration of the tunneling levels will de-

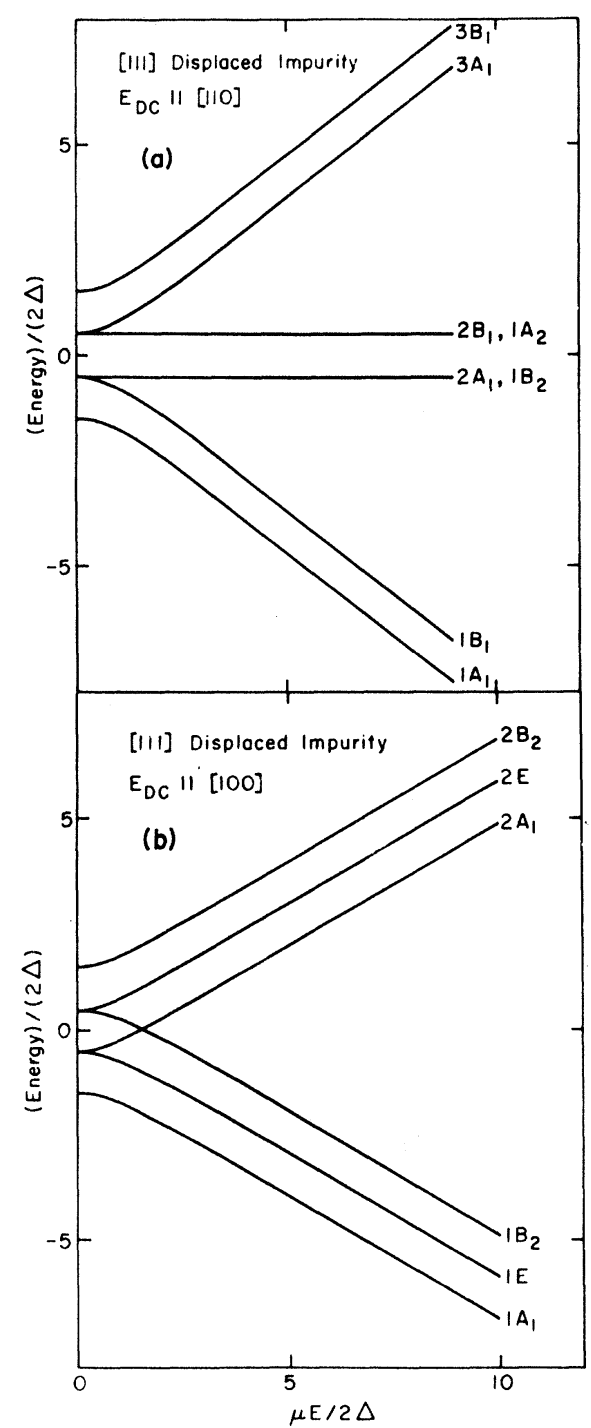

FIG. 9. (a) [110] electric field dependence of the tunneling multiplet for a [111] off-center defect. This assumes only cube-edge tunneling. (b) [100] electric field dependence of the tunneling multiplet for a [111] offcenter defect. This assumes only cube-edge tunneling.

pend strongly on the relative values of these different matrix elements. However, to explain most of the experimental results for $\mathrm{KCl}: \mathrm{Li}^{+}$, it is necessary to allow tunneling only along the cube edge. Thus we take

$$
\begin{aligned}
-\Delta & =\langle a|H| b\rangle=\ldots, \\
0 & =\langle a|H| c\rangle=\langle a|H| g\rangle=\ldots .
\end{aligned}
$$

Then, by degenerate first-order perturbation theory, the Hamiltonian matrix describing the tunneling of the impurity ion in terms of linear combinations of the basis-state eigenfunctions is 


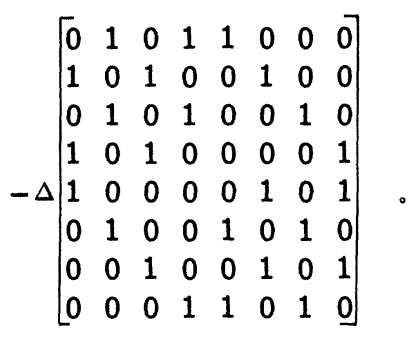

Here, the overlap integrals $\langle a \mid b\rangle,\langle a \mid c\rangle, \ldots$ between the basis states have been neglected. We have also neglected the unperturbed energy $E_{0}$ since it would only give rise to a uniform shift of the resulting energy levels. The diagonalization of this matrix is easily carried out, and we find four equally spaced levels, corresponding to the $A_{1 g}, T_{1 u}, T_{2 g}$, and $A_{2 u}$ irreducible representations of the $O_{h}$ symmetry group. Adjacent levels are separated in energy by an amount $2 \Delta$.

The effect of applying an external electric field can also be calculated. Associated with each of the basis states $|a\rangle,|b\rangle, \ldots$ is an electric dipole moment $\vec{\mu}_{a}, \vec{\mu}_{b}, \ldots$, respectively, resulting from the displacement of the $\mathrm{Li}^{+}$impurity ion from the host-ion cavity center. If the electric field at the impurity site is $\vec{E}$, then the additional energy of the impurity ion when it is in one of the basis states, say, $|a\rangle$, is $\vec{\mu}_{a} \cdot \overrightarrow{\mathrm{E}}$. These contributions must be added to the diagonal elements of the preceding Hamiltonian matrix. The diagonalization of the resulting matrix gives the energy levels as a function of electric field. These results are shown in Figs. 9(a) and 9(b) for [110] and [100] applied fields, respectively. In Table I are listed analytic expressions for the electric field dependences of the energy levels in these two cases.

The cubic symmetry of the lattice-defect system is reduced to a lower symmetry by the electric field. Thus, we expect some transitions which
TABLE I. Analytic expressions for the tunnelinglevel energies as a function of the electric field.

\begin{tabular}{|c|c|}
\hline$[100]$ Field & [110] Field \\
\hline $\begin{aligned} E\left(1 A_{1}\right)= & -2 \Delta \\
& -\Delta\left(1+\frac{\mu^{2} E^{2}}{3 \Delta^{2}}\right)^{1 / 2} \\
E(1 E)= & -\Delta\left(1+\frac{\mu^{2} E^{2}}{3 \Delta^{2}}\right)^{1 / 2} \\
E\left(1 B_{2}\right)= & 2 \Delta-\Delta\left(1+\frac{\mu^{2} E^{2}}{3 \Delta^{2}}\right)^{1 /} \\
E\left(2 A_{1}\right)= & -2 \Delta \\
& +\Delta\left(1+\frac{\mu^{2} E^{2}}{3 \Delta^{2}}\right)^{1 / 2} \\
E(2 E)= & \Delta\left(1+\frac{\mu^{2} E^{2}}{3 \Delta^{2}}\right)^{1 / 2} \\
E\left(2 B_{2}\right)= & 2 \Delta \\
& +\Delta\left(1+\frac{\mu^{2} E^{2}}{3 \Delta^{2}}\right)^{1 / 2}\end{aligned}$ & $\begin{aligned} E\left(2 B_{1}\right)= & +\Delta \\
E\left(1 A_{2}\right)= & +\Delta \\
E\left(3 A_{1}\right)= & -\Delta \\
& +\Delta\left(4+\frac{2 \mu^{2} E^{2}}{3 \Delta^{2}}\right)^{1 / 2} \\
E\left(3 B_{1}\right)= & \Delta+\Delta\left(4+\frac{2 \mu^{2} E^{2}}{3 \Delta^{2}}\right)^{1 / 2}\end{aligned}$ \\
\hline
\end{tabular}

are electric dipole forbidden with no applied field to become optically active in the presence of a field. Also, some transitions which are allowed in zero field may be made weaker by the electric field.

In order to calculate the transition probabilities, we need the electric dipole matrix elements connecting the basis states. In the approximation that has been made, there will only be diagonal elements of the form

$$
\vec{\mu}_{a}=\left\langle a\left|e * \vec{r}_{a}\right| a\right\rangle \text {, }
$$

where $e^{*}$ is the effective charge associated with the $\mathrm{Li}^{+}$ion. Then the transition dipole matrix is

$$
\vec{\mu}=\frac{\left|\vec{\mu}_{a}\right|}{\sqrt{3}}\left[\begin{array}{rr}
(\hat{i}-\hat{j}+\hat{k}) \\
(\hat{i}+\hat{j}+\hat{k}) \\
(-\hat{i}+\hat{j}+\hat{k}) \\
(-\hat{i}-\hat{j}+\hat{k}) \\
(\hat{i}-\hat{j}-\hat{k}) \\
\quad(\hat{i}+\hat{j}-\hat{k}) \\
\quad(-\hat{i}+\hat{j}-\hat{k}) \\
\quad(-\hat{i}-\hat{j}-\hat{k})
\end{array}\right]
$$

The transition probabilities [i.e., $M(E)^{2}$ $\left.=\left|\left\langle\psi_{\text {initial }}|\vec{\mu}| \psi_{\text {final }}\right\rangle\right|^{2}\right]$ have been calculated as a function of [100] electric field. It is found that, with the approximations which were made, transitions which initially were electric dipole forbidden remain forbidden. The transition probabilities for the transitions $1 A_{1} \rightarrow 2 A_{1}, 1 E \rightarrow 2 E$, and $1 B_{2}$ $\rightarrow 2 B_{2}$ decrease according to the equation

$$
M(E)^{2}=\frac{\mu^{2}}{1+\mu^{2} E^{2} / 3 \Delta^{2}}
$$




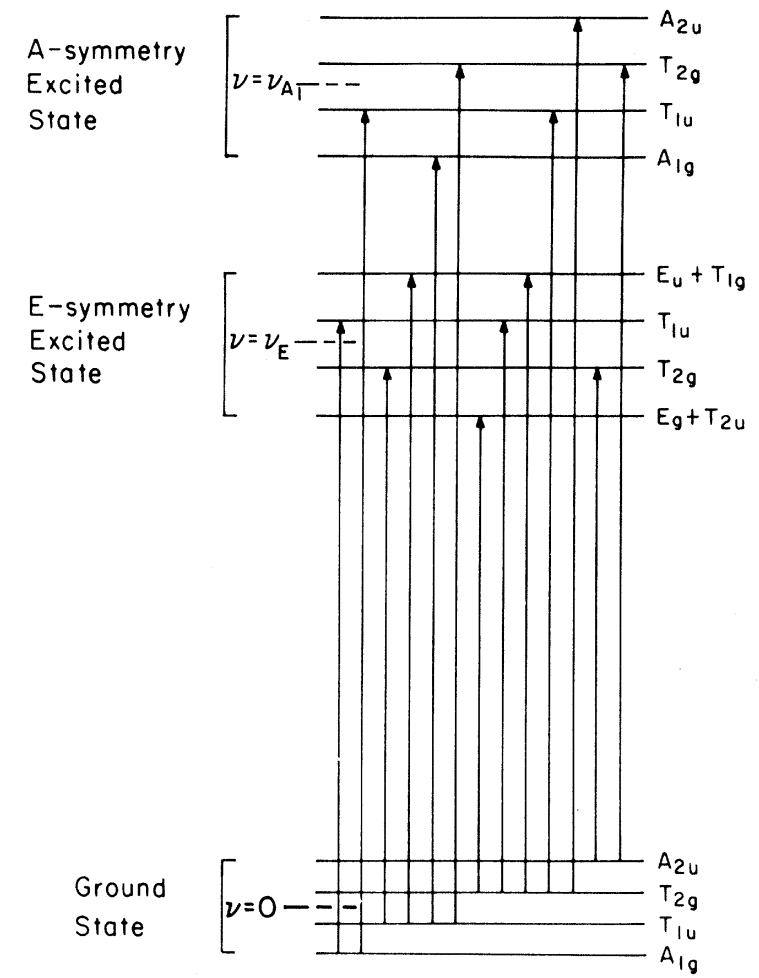

FIG. 10. Energy-level scheme resulting from the extension of the [111] tunneling model to the resonantmode excited states. The arrows indicate the optically active transitions.

The extension of this tunneling calculation to the resonant-mode excited states is straightforward if the small-tunneling-rate approximation is made. If, as for the ground-state calculation, only tunneling along the cube edge is allowed, the calculation gives an energy-level diagram as shown in Fig. 10. Any mixing of levels between the first-excited-state tunneling multiplets and the ground-state and higher-excited-state tunneling multiplets has been neglected. Similarly, tunneling between the basis states of the $A_{1}$ and $E$ first excited states has not been allowed, since this would only lead to mixing of the two $T_{1 u}$ levels. There is no a priori reason why the $A_{1}$ tunneling multiplet should be higher in energy than the $E$ multiplet, but intuitively, this is consistent with the chosen picture in which the potential barriers prohibiting tunneling through the cube-body diagonal are higher than the barriers for tunneling along the cube edge.

It is interesting to note that the tunneling configuration for the $E$ excited state strongly resembles the energy-level configuration resulting from the Devonshire model, ${ }^{40}$ which can be used to describe the motion of a rigid rotor in an octahedral potential with [111] potential minima. This is due to the fact that by allowing only cube-edge tunneling, we have introduced a rotational character into the motion of the impurity ion.

C. Comparison with Experiment

\section{Ground-State Tunneling Multiplet}

Figure 11 (a) shows the tunneling absorption frequency for $\mathrm{KCl}: \mathrm{Li}^{6} \mathrm{Cl}$ as a function of the external [100] electric field. Here, the points are the experimentally observed frequencies and the solid curve is the field dependence calculated from the tunneling model assuming an electric dipole moment $\mu$ of $1.14 e \AA\left(1 e \AA=1.6 \times 10^{-29} \mathrm{~cm}\right.$ ) (uncorrected for local fields) for the $\mathrm{Li}^{6}$ impurities. The data cannot be extended to low enough frequencies to give a reliable value for the zero-field tunneling splitting, so the value $2 \Delta=1.15 \mathrm{~cm}^{-1}$ obtained by Harrison et $a l .{ }^{23}$ was used in fitting the curve.

According to the tunneling model, this absorption line arises from three separate transitions [referring to Fig. 9 (a), the $1 A_{1} \rightarrow 2 A_{1}, 1 E-2 E$, and $1 B_{2} \rightarrow 2 B_{2}$ transitions], all of which have the same transition energy。 Any differences between the three transition frequencies would cause a temperature dependence of the absorption frequency. No such effect was observed outside the experimental uncertainty of $\pm 0.1 \mathrm{~cm}^{-1}$ for temperatures between 1.2 and $4.2^{\circ} \mathrm{K}$. For $E_{\mathrm{IR}} \perp E_{\mathrm{dc}}$, no absorption line was found, in agreement with the predictions of the GBK model.

Figure 11 (b) shows the absorption frequency versus field for a $\mathrm{Li}^{7}$-doped $\mathrm{KCl}$ sample, with $5.0 \times 10^{18} \mathrm{Li}^{*} / \mathrm{cm}^{3}$. The external field was in the [100] direction and the incident radiation was polarized parallel to the field. The solid curve was calculated from the GBK model assuming a zerofield splitting of $2 \Delta=0.8 \mathrm{~cm}^{-1}$ and a $\mathrm{Li}^{7}$ dipole moment of $1.15 e \AA$. This $\mathrm{Li}^{7}$ dipole moment agrees well with that measured for $\mathrm{Li}^{6}$, even though the $\mathrm{Li}^{*}$-impurity concentrations differed by an order of magnitude.

The absorption-frequency-versus-[110]-field data are shown for $E_{\mathrm{IR}} \|$ [110] in Fig. 11 (c) and for $E_{\mathrm{IR}} \|[1 \overline{1} 0]$ in Fig. 11 (d). These two sets of data were taken on the same sample, with a $\mathrm{Li}^{6}$ impurity concentration of $5.0 \times 10^{18} \mathrm{Li}^{6} / \mathrm{cm}^{3}$. For $E_{\text {IR }} \|[110]$, the tunneling model predicts only one absorption line, due to the $1 A_{1} \rightarrow 2 A_{1}$ and $1 B_{1} \rightarrow$ $2 B_{1}$ transitions of Fig. $9(\mathrm{~b})$. (The $1 A_{1}-3 A_{1}$ and $1 B_{1}-3 B_{1}$ transitions are allowed by symmetry, but the tunneling model gives them zero intensity.) Both of these transitions should have the same frequency. There are also other optically active transitions with this frequency, but the initial states for the transitions are not populated at the 


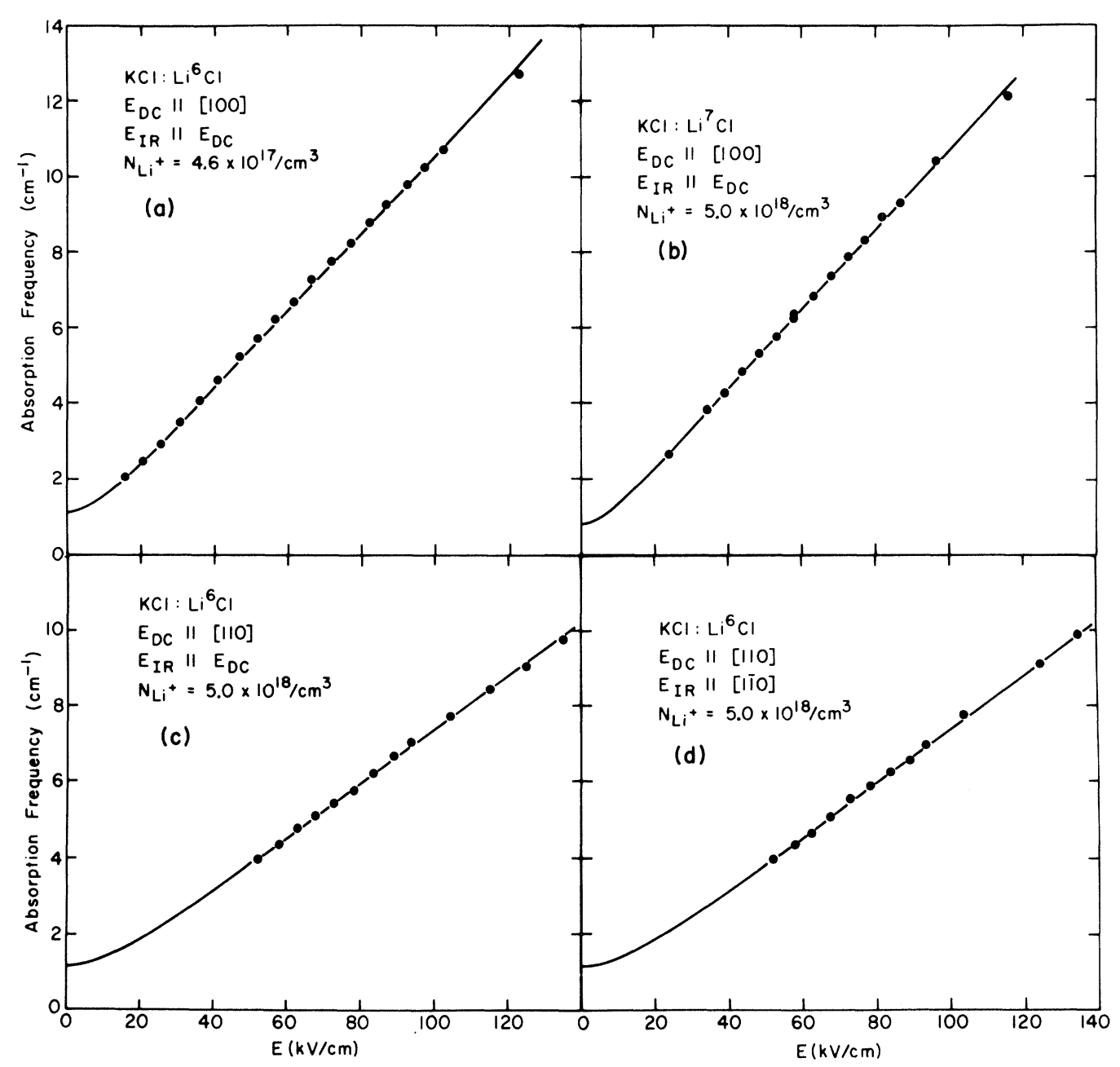

FIG. 11. (a) [100] field dependence of a $\mathrm{KCl}: \mathrm{Li}^{6} \mathrm{Cl}$ tunneling absorption line. These data include points at both 1.2 and $4.2^{\circ} \mathrm{K}$. The solid line is calculated from the GBK model assuming a $\mathrm{Li}^{6}$ dipole moment of $1.14 \mathrm{e} \AA$ and a zero-field tunneling splitting $2 \Delta=1.15 \mathrm{~cm}^{-1}$ 。 (b) [100] field dependence of a $\mathrm{KCl}: \mathrm{Li}^{7} \mathrm{Cl}$ tunneling absorption line. The solid line is calculated from the GBK model assuming a $\mathrm{Li}^{7}$ dipole moment of $1.15 \mathrm{e \AA}$ and a zero-field splitting $2 \Delta=0.8 \mathrm{~cm}^{-1}$. (c) [110] field dependence of a $\mathrm{KCl}: \mathrm{Li}^{6} \mathrm{Cl}$ tunneling absorption line for $E_{\mathrm{I} R} \| E_{\mathrm{dc}}$. The solid line is calculated from the GBK model assuming a $\mathrm{Li}^{6}$ dipole moment of $1.11 e \AA$ and a zero-field splitting $2 \Delta=1.15 \mathrm{~cm}^{-1}$. (d) [110] field dependence of a $\mathrm{KCl}: \mathrm{Li}^{6} \mathrm{Cl}$ tunneling absorption line for $E_{\mathrm{IR}} \|[1 \overline{1} 0]$. The solid line is calculated from the GBK model assuming a $\mathrm{Li}^{6}$ dipole moment of $1.11 e \AA$ and a zero-field splitting of $2 \Delta=1.15 e \AA$.

fields and temperatures used. The solid curve in Fig. 11 (c) is the calculated field dependence assuming a $\mathrm{Li}^{+}$dipole moment of $1.11 e \AA$.

For $E_{\mathrm{IR}} \|$ [110], there are again two allowed transitions which have the same transition energy $\left\lfloor 1 A_{1} \rightarrow 1 B_{2}\right.$ and $1 B_{1} \rightarrow 1 A_{2}$ in Fig. $\left.9(\mathrm{~b})\right]$. The field dependence of these transitions is calculated to be the same as for $E_{\mathrm{IR}} \|[110]$. This is confirmed by the fitted curve in Fig. 11 (d), where again a dipole moment of $1.11 \mathrm{e} \AA$ was found.

It should be pointed out that by considering the polarization dependence of the [100] and [110] electric-field-induced frequency shifts, we have conclusive evidence for a [111] displacement of the
$\mathrm{Li}^{+}$ions in $\mathrm{KCl}$. The electric dipole moment of both $\mathrm{Li}^{6}$ and $\mathrm{Li}^{7}$ impurities is found to be 1.14 $\pm 0.05 e \AA$. This value is in good agreement with that found by other investigators. ${ }^{10,12,16,23}$ It is clear from Fig. 2 that the strength of the absorption line decreases with increasing electric field. As mentioned earlier, the transition probabilities of the allowed transitions are expected to decrease with increasing field. However, to make a numerical comparison between the experiment results and the GBK model, we must first relate the calculated transition probabilities $|M(E)|^{2}$ to the measured absorption strengths $\int_{\text {absorption } 11 \text { ine }} \alpha(\omega) d \omega$. It can be shown ${ }^{41}$ in a straightforward manner that 


$$
\begin{aligned}
\int_{\substack{\text { absorption } \\
\text { line }}} \alpha(\omega) d \omega & =\frac{4 \pi^{2}}{3 c \hbar n} \\
\times & \sum_{\substack{\text { anl } \\
\text { transitions }}} \omega_{i}(E) N_{i}(E)\left|M_{i}(E)\right|^{2},
\end{aligned}
$$

where $\omega_{i}(E)$ is the transition frequency of transition $i$ with an applied electric field $E, N_{i}(E)$ is the number of $\mathrm{Li}^{+}$ions per unit volume in the initial state for transition $i$ with field $E,\left|M_{i}(E)\right|^{2}$ is the transition probability for transition $i$ with field $E$, and $n$ is the index of refraction of the medium.

For $\mathrm{KCl}: \mathrm{LiCl}$ with an external [100] electric field, the sum over all transitions in Eq. (11) includes only the three allowed transitions of Fig. 9 (a), all of which have the same transition energy as a function of field:

$$
\omega_{i}(E)=2 \Delta\left(1+\mu^{2} E^{2} / 3 \Delta^{2}\right)^{1 / 2}
$$

and the same transition probabilities as a function of field, as given by Eq. (10). For the temperatures and applied fields we have used in these measurements, the levels which move down in energy with increasing field will to a very good approximation be the only levels which are populated. So we take $N_{i}=N=$ total number of $\mathrm{Li}^{+}$ions per unit volume. Then we have

$$
\int \alpha(\omega) d \omega=\frac{4 \pi^{2}(2 \Delta) N \mu^{2}}{3 c \hbar n\left(1+\mu^{2} E^{2} / 3 \Delta^{2}\right)^{1 / 2}} .
$$

Figure 12 shows the measured absorption strength $\int \alpha(\omega) d \omega$ as a function of a [100] external electric field. The sample was doped with 4.6 $\times 10^{17} \mathrm{Li}^{6} / \mathrm{cm}^{3}$. For an applied field of $100 \mathrm{kV} / \mathrm{cm}$, the measured absorption strength is $0.15 \mathrm{~cm}^{-2}$. For a Lorentz local-field correction of $2.17, \mu$ $=(1.14 / 2.17) e \AA$. Taking $2 \Delta=1.15 \mathrm{~cm}^{-1}, n=2.12$, and $E=100 \mathrm{kV} / \mathrm{cm}, E q$. (13) predicts that

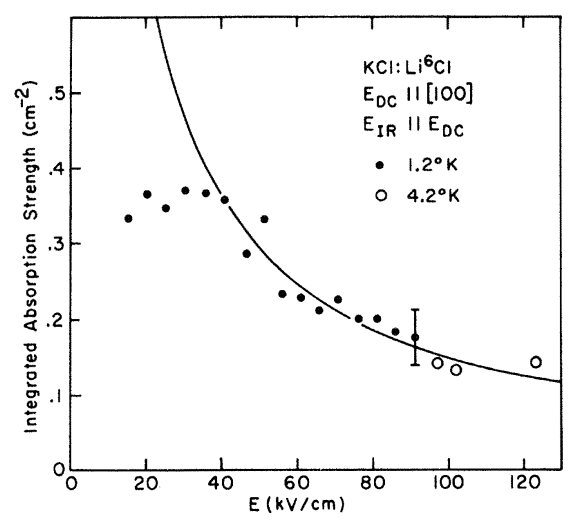

FIG. 12. [100] field dependence of the $\mathrm{KCl}: \mathrm{Li}^{6} \mathrm{Cl}$ tunneling absorption strength.

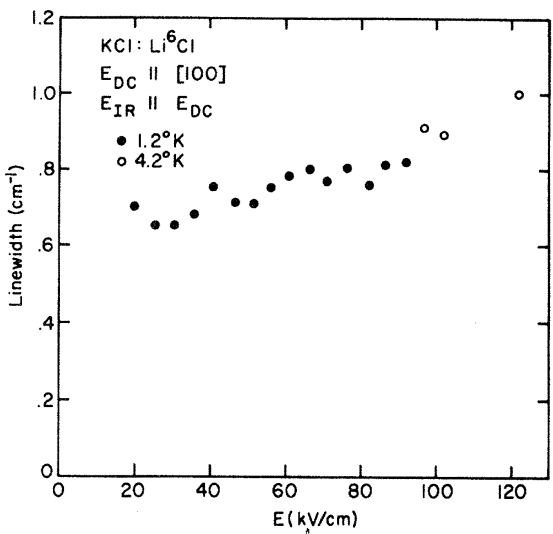

FIG. 13. [100] field dependence of the $\mathrm{KCl}: \mathrm{Li}^{6} \mathrm{Cl}$ tunneling absorption linewidth.

$$
\int \alpha(\nu) d \nu=0.072 \mathrm{~cm}^{-2} \text {. }
$$

Thus the predicted absorption strength at high fields is a factor of 2 smaller than the measured absorption strength. This is considered to be reasonable agreement in view of the uncertainty in the absolute value of the measured absorption strength for our rather complicated sample geometry. Also, there is evidence ${ }^{23}$ that a considerable portion of the $\mathrm{Li}^{+}$impurities do not participate in the tunneling motion but are condensed into another configuration. Such an aging effect would reduce the measured absorption strength.

The solid line in Fig. 12 is given by Eq. (13), scaled by a factor of $\frac{1}{2.2}$. It fits the experimental data well for applied fields above $35 \mathrm{kV} / \mathrm{cm}$. Below this field there is some overlap between the absorption line in zero field and the line with field applied. This effect reduces the measured absorption strength.

The width of the absorption line exhibits only a weak field dependence, as shown in Fig. 13 for a [100] field and $4.6 \times 10^{17} \mathrm{Li}^{6} / \mathrm{cm}^{3}$. At least part of the broadening at high fields is due to the inhomogeneity of the applied field. There may also be lifetime broadening because of the higher phonon density of states at higher frequencies. However, the measured phonon relaxation time in low fields of $10^{-8} \mathrm{sec}^{13}$ accounts only for a width of $\sim 5 \times 10^{-4}$ $\mathrm{cm}^{-1}$.

The linewidths of the tunneling absorptions depend strongly on the $\mathrm{Li}^{+}$-impurity concentration. For $4.6 \times 10^{17} \mathrm{Li}^{+} / \mathrm{cm}^{3}$, the linewidth at low-field values was $0.7 \mathrm{~cm}^{-1}$. For $5 \times 10^{18} \mathrm{Li}^{+} / \mathrm{cm}^{3}$, the linewidth was about $2 \mathrm{~cm}^{-1}$. One contribution to the linewidth must come from the random electric fields caused by the presence of the dipolar $\mathrm{Li}^{+}$ impurities. We can obtain a rough estimate of this contribution simply, following Bron and Drey- 
fus. ${ }^{13}$ They have shown that the average magnitude of the random internal electric fields due to the presence of dipolar impurities is given approximately by

$$
\bar{E}=3.2 \mu / \epsilon r^{3},
$$

where $r$ is the average separation of the impurity ions, $\epsilon$ is the dielectric constant, and $\mu$ is the impurity dipole moment.

It should be pointed out that for such large concentrations of $\mathrm{Li}^{+}$ions as have been used in this investigation, the $\mathrm{KCl}: \mathrm{LiCl}$ samples cannot be treated as simple dielectrics, since the polarizability of the $\mathrm{Li}^{+}$ions does not have a linear dependence on electric field. ${ }^{7}$ For this rough calculation, we neglect this effect and take $\epsilon=\epsilon$ (pure $\mathrm{KCl})$. For $\mathrm{KCl}$ with $10^{18} \mathrm{Li}^{+} / \mathrm{cm}^{3}, \epsilon=4.5, \mu=1.14$ $e \AA$, and $r=10^{-6} \mathrm{~cm}$, so that $E=1.2 \mathrm{kV} / \mathrm{cm}$. Thus, for $10^{18} \mathrm{Li}^{+} / \mathrm{cm}^{3}$, the dipolar line broadening should correspond to approximately $\pm 1.2 \mathrm{kV} / \mathrm{cm}$. Since the absorption line shifts by about $1 \mathrm{~cm}^{-1}$ for a $10-\mathrm{kV} / \mathrm{cm}$ increase in the applied electric field, we see that for $10^{18} \mathrm{Li}^{+} / \mathrm{cm}^{3}$, the dipolar contribution to the linewidth should be $0.24 \mathrm{~cm}^{-1}$. This contribution depends linearly on the $\mathrm{Li}^{+}$concentration, so that for $4.6 \times 10^{17} \mathrm{Li}^{+} / \mathrm{cm}^{3}$, the dipolar broadening is $0.1 \mathrm{~cm}^{-1}$. This is significantly less than the observed linewidth of $0.7 \mathrm{~cm}^{-1}$, and indicates the presence of other broadening mechanisms, such as inhomogeneous lattice strains. ${ }^{16,42}$ In support of this, paraelectric resonance experiments at $64 \mathrm{GHz}$ on samples of still lower $\mathrm{Li}^{+}$ concentrations $\left(6 \times 10^{16} \mathrm{Li}^{7} / \mathrm{cm}^{3}\right)$ by Herendeen and Silsbee ${ }^{16}$ indicate a linewidth of $0.5 \mathrm{~cm}^{-1}$ for the tunneling transitions. This is in good agreement with the low-concentration linewidths presented here. Also, the observed $1.3-\mathrm{cm}^{-1}$ increase in linewidth upon increasing the $\mathrm{Li}^{+}$concentration from $4.6 \times 10^{17}$ to $5 \times 10^{18} \mathrm{Li}^{+} / \mathrm{cm}^{3}$ agrees very well with the $1.2-\mathrm{cm}^{-1}$ increase predicted by dipolar broadening.

\section{Resonant-Mode Excited States}

Gome $z^{43}$ has extended the GBK model to the $\mathrm{KCl}$ : LiCl excited-state multiplet, assuming locally harmonic potential wells for the $\mathrm{Li}^{+}$ion. In this case, there are only two independent parameters, namely, $\omega$, the spherical harmonic oscillator frequency describing the local potential wells, and $\delta$, the $\mathrm{Li}^{+}$displacement in the [111] direction. Letting $\omega=41.7 \mathrm{~cm}^{-1}$, Gomez calculated the excited-state energy levels as a function of $\delta$, which he varied between 0.82 and $1.4 \AA$. He found the following results:

(i) If the model is to fit experiment, the observed $40-\mathrm{cm}^{-1}$ absorption band must be the envelope of
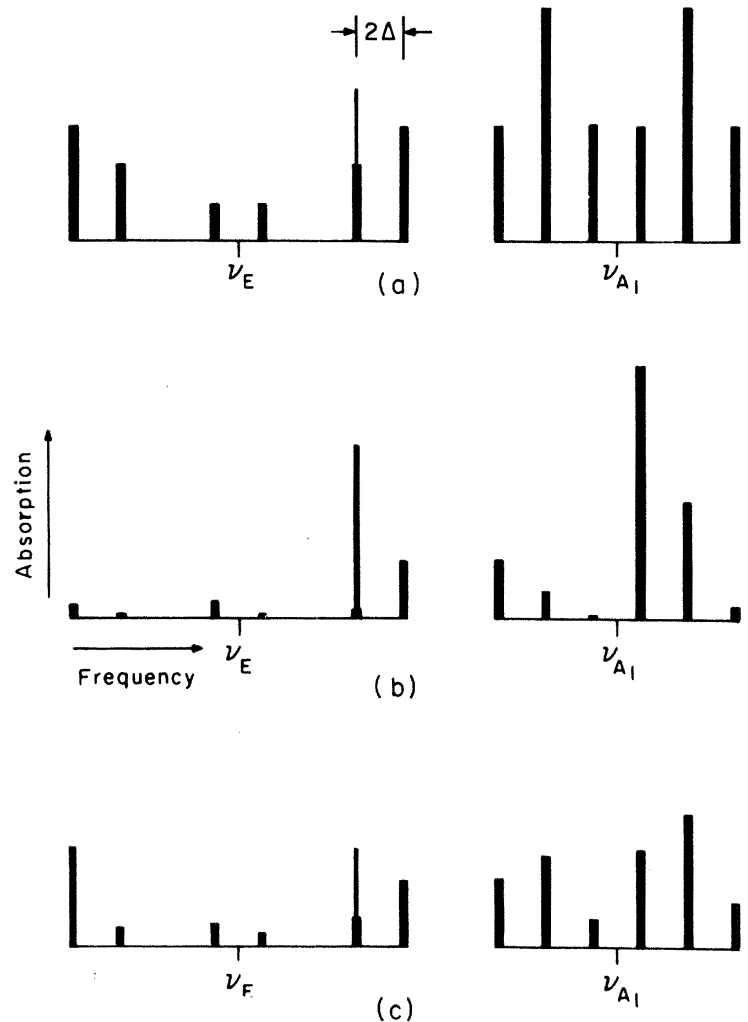

FIG. 14. (a) $\mathrm{KCl}: \mathrm{Li}^{6} \mathrm{Cl}$ tunneling-model absorption spectrum. This shows the absorption frequencies for the $A_{1}$ and $E$ excited-state tunneling multiplets, assuming the excited-state tunneling splittings to be twice the ground-state tunneling splittings. The height of each line is proportional to the calculated transition probability. (b) $\mathrm{KCl}: \mathrm{Li}^{6} \mathrm{Cl}$ tunneling-model absorption spectrum, as in Fig. 14(a), but the transition probabilities have been scaled by the Boltzmann population at $1.15^{\circ} \mathrm{K}$ of the initial state for the transition. (c) $\mathrm{KCl}: \mathrm{Li}^{6} \mathrm{Cl}$ tunneling-model absorption spectrum, as in Fig. 14 (a), but the transition probabilities have been scaled by the Boltzmann population at $4.2^{\circ} \mathrm{K}$ of the initial state for the transition.

many closely spaced absorption lines.

(ii) The centroid of the $40-\mathrm{cm}^{-1}$ band should move up in frequency as the temperature is lowered from $4.2{ }^{\circ} \mathrm{K}$.

(iii) The half-width of the $40-\mathrm{cm}^{-1}$ band should be approximately $5-10 \mathrm{~cm}^{-1}$.

(iv) The centroid of the $\mathrm{Li}^{6}$ absorption band should be higher in frequency than the $\mathrm{Li}^{7}$ absorption band by $2-4 \mathrm{~cm}^{-1}$, depending on the value of $\delta$ and the temperature.

We see that Gomez's calculations agree with experiment on points (i) and (ii), but disagree on points (iii) and (iv). These last two points will require closer scrutiny for possible explanations.

In Fig. 10, we showed the energy-level scheme which would result if the local potential wells for the $\mathrm{Li}^{+}$ion were not spherical, but exhibited $C_{3 v}$ 
symmetry. We now consider this in more detail.

Taking the tunneling splitting in both the $A_{1}$ and $E$ excited-state manifolds to be approximately twice the tunneling splitting in the ground-state manifold, we get the transition spectrum shown in Fig. 14 (a). In each case, the height of the line represents the transition probability. Within the framework of this model, this shows the approximate frequency spectrum from which we must try to construct the experimental results. The variable quantities in this picture are $\omega_{E}, \omega_{A_{1}}$, and the tunneling splittings. $\omega_{E}$ and $\omega_{A 1}$ are expected to increase by about the factor $\left(\frac{7}{6}\right)^{1 / 2}$ when $\mathrm{Li}^{6}$ is substituted for $\mathrm{Li}^{7}$, if the potential well can be considered to be harmonic. In principle, this restriction will be weakened if the potential energy is less strongly dependent on the impurity displacement than it would be for a harmonic well, or if there is some effective-mass contribution to the impurity mass from the neighboring host lattice ions. In any case, it is expected that $\omega_{E}$ and $\omega_{A_{1}}$ will be greater for $\mathrm{Li}^{6}$ than for $\mathrm{Li}^{7}$. At the same time, the tunneling splittings are expected to be larger for $\mathrm{Li}^{6}$ than for $\mathrm{Li}^{7}$. This effect will tend to spread the $\mathrm{Li}^{6}$ absorption over a wider frequency range than the $\mathrm{Li}^{7}$ absorption lines.

For comparison with the experimental results, the calculated transition probabilities must be scaled by the population of the initial state for the transition. This has been done for $\mathrm{KCl}: \mathrm{Li}^{6} \mathrm{Cl}$ at temperatures of 1.15 [Fig. 14 (b)] and $4.2^{\circ} \mathrm{K}$ [Fig. 14 (c)]. We first note that for both the $A_{1}$ and $E$ tunneling multiplets, the absorption is predicted to be stronger on the high-frequency side of the centroid frequency than on the low-frequency side. This is not in agreement with experiment (see Figs. 4 and 5). Also, the model predicts that the low-frequency side of the absorption will be stronger at 4.2 than at $1.15^{\circ} \mathrm{K}$, with the high-frequency side having the opposite temperature dependence. It is found experimentally that the strength of the high-frequency side of the absorption band remains approximately constant for these two temperatures. Furthermore, it does not seem possible to reproduce even roughly the observed line shapes by adjusting the relative positions of $\omega_{A_{1}}$ and $\omega_{E}$.

It was noted earlier that the $\mathrm{Li}^{6}$ and $\mathrm{Li}^{7}$ absorption linewidths are nearly identical. This too is inconsistent with the model. For the ground-state tunneling multiplet the $\mathrm{Li}^{6}$ tunneling splitting is observed to be $40 \%$ larger than the $\mathrm{Li}^{7}$ tunneling splitting. If this were also true for the excitedstate multiplets, a rather large difference in linewidth would be expected for the two isotopes.

This model also fails to explain the negative isotope shift. First, $\omega_{E}$ and $\omega_{A_{1}}$ are expected to be greater for $\mathrm{Li}^{6}$ than for $\mathrm{Li}^{7}$. Second, since the absorption strength is predicted to be greater on the high-frequency side of the line, and since the $\mathrm{Li}^{6}$ tunneling splitting is predicted to be greater than that for $\mathrm{Li}^{7}$, the net result will be a positive isotope shift.

It is apparent that this model does not explain quantitatively the $\mathrm{KCl}: \mathrm{Li}^{+}$excited-state absorption spectrum. Qualitatively, it can be seen that the agreement between the model and experiment would improve considerably if the absorption strengths of the (predicted) higher-frequency transitions were made weak relative to the lower-frequency transitions. This would roughly explain the shape, the temperature dependence, and the width of the absorption. Also, the larger downward tunneling splitting for $\mathrm{Li}^{6}$ would tend to cancel out the increase in $\omega_{E}$ and/or $\omega_{A_{1}}$, and hence reduce the isotope shift.

At this point, it is worth noting the results of a calculation by Benedek, ${ }^{44}$ in which he considered the effects of third- and fourth-order anharmonicity on the $\mathrm{KCl}: \mathrm{Li}^{+} 40-\mathrm{cm}^{-1}$ band. In particular, he found that the third-order anharmonic coupling of the defect resonant mode with itself due to the lack of inversion symmetry at each off-center equilibrium site may be large enough to result in $\bar{\omega}\left({ }^{6} \mathrm{Li}^{+}\right)$being smaller than $\bar{\omega}\left({ }^{7} \mathrm{Li}^{+}\right)$.

Another approach is to use the Devonshire model to describe the $40-\mathrm{cm}^{-1}$ band, as suggested by Harrison et al. ${ }^{23}$ The Devonshire model is equivalent to the resonant-mode model if we consider only the energy levels in the $\omega_{E}$ excited-state multiplet, presumably by assuming $\omega_{A_{1}} \gg \omega_{E}$. The use of the Devonshire model has an advantage in that it can take into account the changes in transition probability which occur because the excited state cannot be considered to be in the extreme tunneling limit. Qualitatively, this means that transitions in the Devonshire model which extrapolate back to $\Delta J= \pm 1$ transitions for the free rotor will be enhanced at the expense of those symmetry-allowed transitions for which $|\Delta J|>1$.

This in effect increases the absorption strength in the low-frequency region of the $\omega_{E}$ spectrum shown in Fig. 14, and so improves the agreement of the line shape with experiment. It also tends to make the isotope shift smaller, but does not make it negative. Harrison et al. calculated an isotope shift of $\bar{\omega}\left({ }^{6} \mathrm{Li}^{+}\right) / \bar{\omega}\left({ }^{7} \mathrm{Li}^{+}\right)=1.04$; but, they included only one of the four $\Delta J= \pm 1$ allowed transitions in their calculation. The calculated shift is increased slightly from this value if all allowed transitions are considered. In addition, the Devonshire model does not explain why the measured linewidths for ${ }^{6} \mathrm{Li}^{+}$and ${ }^{7} \mathrm{Li}^{+}$impurities are nearly identical, and it fails to reproduce some features 
of the temperature dependence. Still, it does qualitatively improve the agreement with experiment, and may be a more accurate representation of the $\mathrm{KCl}: \mathrm{Li}^{+}$system than our resonant-mode model.

\section{IV. $\mathrm{RbCl}: \mathrm{AgCl}$}

\section{A. Experimental Results}

Over the past three years some rather strong evidence that $\mathrm{Ag}^{+}$impurities occupy an off-center position in $\mathrm{RbCl}$ has been presented. Dreybrodt and Fussgaenger ${ }^{45}$ have pointed out that displaced $\mathrm{Ag}^{+}$impurities can explain some features of the temperature dependence of the $\mathrm{Ag}^{+} \mathrm{uv}$ absorption band in $\mathrm{RbCl}$. Nolt ${ }^{25}$ has measured the far-infrared absorption spectrum of $\mathrm{RbCl}: \mathrm{Ag}^{+}$, finding three absorption lines between 21 and $37 \mathrm{~cm}^{-1}$. For an applied [100] uniaxial stress, and the electric field vector of the incident radiation polarized parallel to the stress, Nolt found that absorption lines at 21.3 and $26.5 \mathrm{~cm}^{-1}$ decreased in strength, while the remaining absorption line at $36.7 \mathrm{~cm}^{-1}$ increased in strength. Such stress-induced dichroisms are also consistent with an off-center defect.

More recently, Kapphan and Luty ${ }^{12}$ have observed an electrocaloric effect in $\mathrm{RbCl}: \mathrm{Ag}^{+}$. Measuring the amount of paraelectric cooling in the high-field (saturation) region for both [100] and [111] applied electric fields, they concluded that the $\mathrm{Ag}^{+}$ions are off center in the [111] direction with a dipole moment of $0.83 \pm 0.08 e \AA$.

The $\mathrm{RbCl}: \mathrm{AgCl}$ far-infrared absorption spectrum is shown in Fig. 15 for a sample containing 164-ppm Ag by weight at a temperature of $1.2{ }^{\circ} \mathrm{K}$. There are three absorption lines of roughly comparable absorption strengths at 21.3, 26.5, and $36.7 \mathrm{~cm}^{-1}$. High-resolution measurements $\left(0.09 \mathrm{~cm}^{-1}\right)$ on the two lower-frequency lines have shown no fine structure. The absorption spectrum at $4.2{ }^{\circ} \mathrm{K}$ is identical to that of Fig. 15 to within

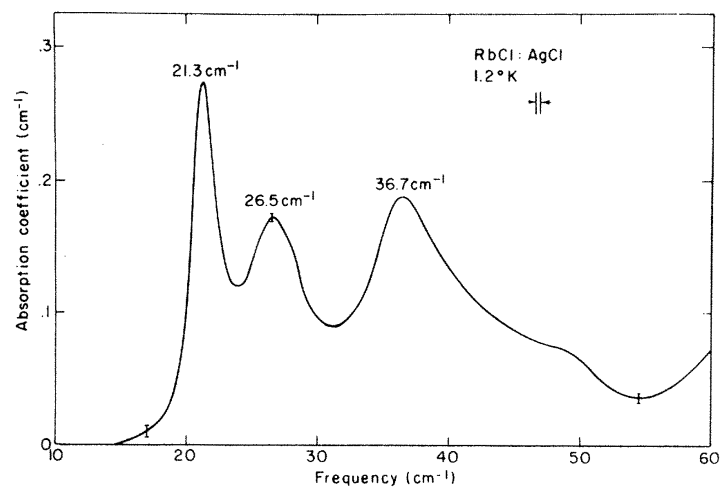

FIG. 15. $\mathrm{RbCl}: \mathrm{AgCl}$ absorption spectrum. the indicated noise level. These two facts suggest that the tunneling levels are closely spaced compared to $1 \mathrm{~cm}^{-1}$.

Some preliminary [100] electric field measurements have been made on this system. For $E_{\mathrm{IR}}$ $\| E_{\mathrm{dc}}$, the 21.3- and $26.5-\mathrm{cm}^{-1}$ lines increase in absorption strength with increasing field, while the $36.7-\mathrm{cm}^{-1}$ line decreases in strength. For $E_{\mathrm{IR}}$ $\perp E_{\mathrm{dc}}$, the opposite results are found; the two lower-frequency lines decrease in strength with increasing field, and the $36.7-\mathrm{cm}^{-1}$ line increases in strength. These dichroisms are all very weak, being less than $\sim 5 \%$ of the zero-field absorption strength even for electric fields greater than 100 $\mathrm{kV} / \mathrm{cm}$. This is in contrast to the stress dichroisms observed by Nolt, where changes in strength of more than a factor of 2 were observed. These absorption lines shifted slightly in the presence of a field, but the shifts were too small relative to the linewidths for any quantitative analysis.

We also attempted to observe a field-induced splitting of the low-lying tunneling levels, but no absorption lines were found. As will be shown, this is consistent with a small zero-field tunneling splitting.

\section{B. Discussion}

There are several unreconciled differences between the results presented here and the electrocaloric measurements of Kapphan and Luty. ${ }^{12}$ First, the presence of only small electric-fieldinduced shifts and dichroisms in the far-infrared absorption spectrum indicates a small dipole moment for the $\mathrm{Ag}^{+}$ions, whereas Kapphan and Luty found a rather large dipole moment.

Second, our experimental results on $\mathrm{RbCl}: \mathrm{Ag}^{+}$ cannot be interpreted in terms of a simple [111] displacement of the $\mathrm{Ag}^{+}$ions. In the limit of small tunneling splittings for both the resonantmode ground and excited states, only two widely separated absorption lines are expected for a [111] defect. The separation of the three observed absorption lines appear to be too great to be due to tunneling in the resonant-mode excited state.

The fact that no low-frequency absorption lines were observed in the presence of an electric field is not too surprising. Assuming a small groundstate tunneling splitting of $2 \Delta=0.1 \mathrm{~cm}^{-1}$ and a dipole moment $\mu=0.83 e \AA$, we can use Eq. (13) to calculate the absorption strength expected for a [111] defect. For the measured Ag concentration of $2.5 \times 10^{18} \mathrm{Ag} / \mathrm{cm}^{3}$, and an applied field of 100 $\mathrm{kV} / \mathrm{cm}$, Eq. (13) gives

$$
\int \alpha(\omega) d \omega=0.004 \mathrm{~cm}^{-2} \text {. }
$$

Such a small integrated absorption strength would not be observable for the thickness samples used 
$(\sim 2 \mathrm{~cm})$.

We will now show that the far-infrared experimental results on $\mathrm{RbCl}: \mathrm{Ag}^{+}$are consistent with a [110] dipole orientation for the $\mathrm{Ag}^{+}$impurities. It has been pointed out that the tunneling splitting of the $\mathrm{Ag}^{+}$vibrational ground state is small. Then even in the presence of small electric fields, we may ignore tunneling and work in the high-temperature (classical) approximation. In this picture, the $\mathrm{Ag}^{+}$ion has 12 equivalent equilibrium sites in the [110] directions. In each of these sites the vibrational motion of the impurity occurs in a local potential well of $C_{2 v}$ symmetry, so that the first excited state consists of three levels, corresponding to the $A_{1}, B_{1}$, and $B_{2}$ irreducible representations of the $C_{2 v}$ symmetry group. The optically active transitions between the groundstate and these three excited-state levels are assumed to give rise to the three observed absorption lines. A schematic representation of the equilibrium sites and of the three nondegenerate excited-state vibrations is shown in Fig. 16. $\omega_{1}$, $\omega_{2}$, and $\omega_{3}$ are the three observed absorption frequencies. The associated arrows indicate the three independent directions of charge motion. It should be noted that of the three simple off-center displacement directions ([100], [110], and [111]), only a [110] displacement is consistent with three absorption lines in the classical limit.

Associated with each of the three absorption frequencies $\omega_{1}, \omega_{2}$, and $\omega_{3}$ are transition dipoles $\vec{\mu}_{1 k}, \vec{\mu}_{2 k}$, and $\vec{\mu}_{3 k}$, respectively. Here, the subscript $k$ refers to one of the 12 equilibrium sites. The transition probability at frequency $\omega_{i}$ for an impurity in site $k$ is then proportional to the square of the component of $\vec{\mu}_{i k}$ in the direction of the $E$ vector of the incident radiation. In the absence of an external electric field, the $\mathrm{Ag}^{+} \mathrm{im}-$ purities spend an equal amount of time in each of the 12 equilibrium sites, so that the absorption strengths of $\omega_{1}, \omega_{2}$, and $\omega_{3}$ will be in the ratios $\left|\vec{\mu}_{1}\right|^{2}:\left|\vec{\mu}_{2}\right|^{2}:\left|\vec{\mu}_{3}\right|^{2}$.

If an external electric field is applied in the $+z$ direction, the $\mathrm{Ag}^{+}$ions will tend to be localized in the four sites lying in the $+z$ plane. If all of the $\mathrm{Ag}^{+}$ions were localized in these sites, the transition probabilities for $\omega_{1}, \omega_{2}$, and $\omega_{3}$ would be in the ratios $0:\left|\vec{\mu}_{2}\right|^{2}:\left|\vec{\mu}_{3}\right|^{2}$ for $E_{\mathrm{IR}} \| E_{\mathrm{dc}}$ and $2\left|\vec{\mu}_{1}\right|^{2}$ : $\left|\vec{\mu}_{2}\right|^{2}:\left|\vec{\mu}_{3}\right|^{2}$ for $E_{\mathrm{IR}} \perp E_{\mathrm{dc}}$. Thus for $E_{\mathrm{IR}} \| E_{\mathrm{dc}}$, the trend is for one line $\left(\omega_{1}\right)$ to grow weaker with increasing electric field, and the other two lines $\left(\omega_{2}\right.$ and $\left.\omega_{3}\right)$ to grow stronger. To fit the experimental results, we must assign $\omega_{1}=36.5 \mathrm{~cm}^{-1}$. $\omega_{2}$ and $\omega_{3}$ cannot be assigned unambiguously. For $E_{\mathrm{IR}} \perp E_{\mathrm{dc}}$, then the $36.5-\mathrm{cm}^{-1}$ line should grow stronger with increasing electric field, and the other two should grow weaker. This is observed

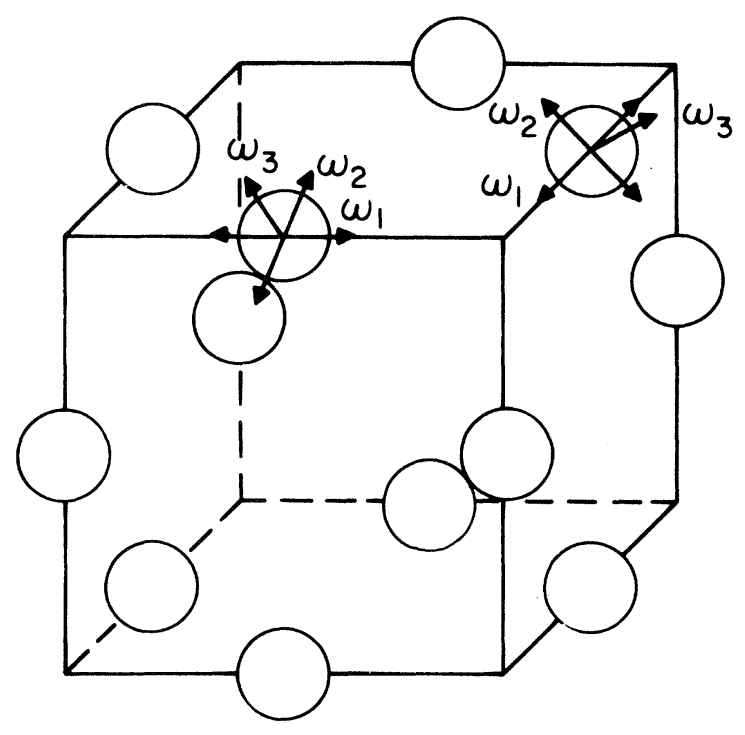

FIG. 16. Equilibrium sites and resonant-mode excited-state basis states for a [110] off-center impurity.

experimentally, so that the electric-field-induced dichroisms predicted by a [110] off-center position are consistent with the experimental results, although $\omega_{2}$ and $\omega_{3}$ still cannot be unambiguously assigned.

Similarly, Nolt's stress results are consistent with this picture. With an applied uniaxial stress in the $z$ direction, the $\mathrm{Ag}^{+}$ions will tend to be localized in the $z=0$ plane of the off-center sites. For radiation polarized parallel to the stress, this will cause the line at $\omega_{1}=36.5 \mathrm{~cm}^{-1}$ to grow stronger and $\omega_{2}$ and $\omega_{3}$ to grow weaker, in agreement with experiment.

The electrocaloric measurements of Kapphan and Luty are not, however, consistent with a simple [110] displacement of the $\mathrm{Ag}^{+}$ions. They measured the ratio of the saturation cooling for [100] and [111] external electric fields. For a [111] defect, this ratio is calculated to be

$$
\Delta T(\lfloor 100]) / \Delta T(\lfloor 111])=0.33,
$$

in reasonable agreement with their experimental results. For a [110] defect, this same ratio is calculated to be 0.82 .

Thus, it appears that in order to explain both the electrocaloric results and the far-infrared results, another approach must be taken. One possibility is that the $\mathrm{Ag}^{+}$ions see potential-energy minima in both the [110] and [111] directions. An analysis of this situation would require a knowledge of the relative depths of the two inequivalent potential wells as well as both of the associated electric dipole moments. However, with the appropriate values of these parameters, such a 
model could likely explain both sets of experimental results.

Ancther explanation that cannot be ruled out is that the $\mathrm{Ag}$ impurities form more than one type of defect center, and that the electrocaloric measurements probe primarily one impurity configuration and the far-infrared measurements probe another.

In either case, it is apparent that more experiments will be necessary if the $\mathrm{RbCl}: \mathrm{Ag}^{+}$system is to be understood in detail.

\section{V. $\mathrm{NaCl}: \mathrm{NaOH}$}

\section{A. Experimental Results}

\section{Temperature Dependence}

Far-infrared transmission measurements on the $\mathrm{OH}^{-}$-doped $\mathrm{NaCl}$ samples were made over the frequency range 1.5 to $100 \mathrm{~cm}^{-1}$. The absorption spectrum for $60-\mathrm{ppm} \mathrm{OH}^{-}$impurities is shown in Fig. 17 for temperatures between 0.6 and $4.1^{\circ} \mathrm{K}$. For transmission measurements at $0.6^{\circ} \mathrm{K}$, the $\mathrm{He}^{3}$-sample cryostat described in Sec. II was used to cool both the pure and doped $\mathrm{NaCl}$ samples. Because of the problem of radiation leaking through the sample covered by the shutter, measurements of the 1.2 to $0.6{ }^{\circ} \mathrm{K}$ temperature-induced absorption coefficient were made, as described in Sec. II. Even with this correction, however, the $\alpha$ scale for the $0.6^{\circ} \mathrm{K}$ data in Fig. 17 is only accurate to within a factor of about 1.1 .

We see that the temperature dependence of some of the absorption lines is extremely rapid. The absorption lines at 9.3, 10.2 (observable as a shoulder of the $9.3-\mathrm{cm}^{-1}$ line), and $22 \mathrm{~cm}^{-1}$ are all very prominent at $4.1^{\circ} \mathrm{K}$. At $0.6^{\circ} \mathrm{K}$, there is essentially no absorption at these frequencies. The $12.2-\mathrm{cm}^{-1}$ absorption line also decreases in

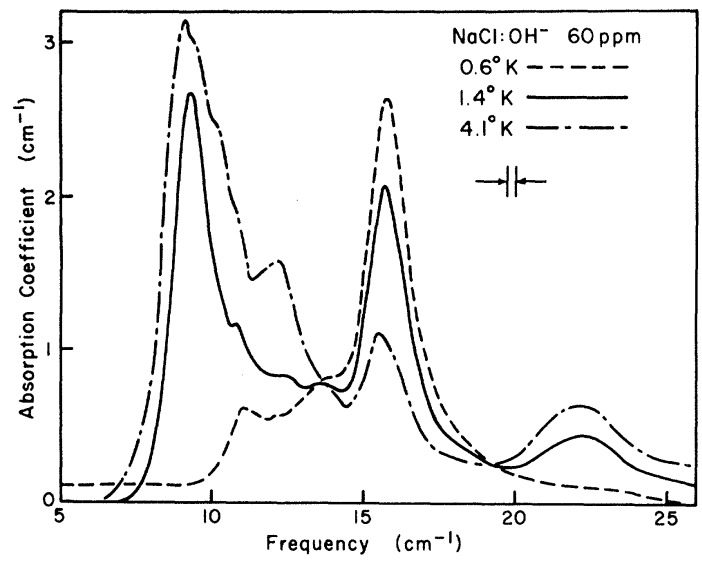

FIG. 17. Temperature dependence of the $\mathrm{NaCl}: \mathrm{OH}^{-}$ absorption spectrum.

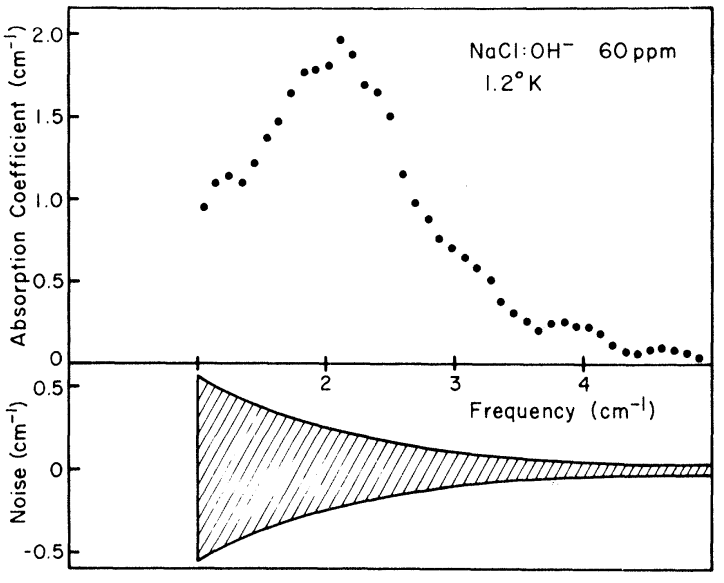

FIG. 18. $\mathrm{NaCl}: \mathrm{OH}^{-}$tunneling absorption line.

strength with decreasing temperature. The 15.7$\mathrm{cm}^{-1}$ absorption line has just the opposite temperature dependence: It increases in strength with decreasing temperature. It is difficult to determine the temperature dependence of the remaining structure between 10 and $15 \mathrm{~cm}^{-1}$ because of the overlapping absorption of adjacent lines, and possibly because of other absorptions which cannot be distinguished from the background.

For there to be such a rapid temperature dependence, the $\mathrm{OH}^{-}$impurities must have motional energy states which are within about $3 \mathrm{~cm}^{-1}$ of the ground-state energy level. Furthermore, the $15.7-\mathrm{cm}^{-1}$ absorption line must arise from a transition for which the initial state is the ground state, or at least is a level close to the groundstate level compared to $0.6^{\circ} \mathrm{K}\left(0.4 \mathrm{~cm}^{-1}\right)$. The 9.3-, 10.2-, 12.2-, and $22-\mathrm{cm}^{-1}$ lines must arise from levels higher in energy than the ground state.

In order to confirm the presence of such lowlying levels, the absorption of $\mathrm{NaCl}: \mathrm{OH}^{-}$was measured down to $1.5 \mathrm{~cm}^{-1}$ with the lamellar interferometer. These results are shown in Fig. 18. The data show a broad peak in absorption at about $2 \mathrm{~cm}^{-1}$ for a sample temperature of $1.2^{\circ} \mathrm{K}$. However, because of the relatively high noise level at such low frequencies (see the shaded area in Fig. 18), the absorption peak may not be exactly where it is shown, and there may be additional structure. Furthermore, the scale of the absorption coefficient may be as much as $50 \%$ too large. The reason for this is that the output of the mercury-arc source at $2 \mathrm{~cm}^{-1}$ is less than $5 \%$ of the output at $20 \mathrm{~cm}^{-1}$. Thus a small error in the zero-transmission level will give rise to large (and approximately uniform) errors in the absolute value of the absorption. However, the important 
point here is that there are energy levels about $2-\mathrm{cm}^{-1}$ apart.

In addition to the absorption shown in Figs. 17 and 18, a broad and extremely weak absorption peak was observed at about $35 \mathrm{~cm}^{-1}$. This is superimposed on a smooth background absorption which flattens off at about $44 \mathrm{~cm}^{-1}$. There is also $\mathrm{OH}^{-}$-induced absorption above $80 \mathrm{~cm}^{-1}$ which increases monotonically to well above $100 \mathrm{~cm}^{-1}$. These absorptions however were not studied in detail.

We also attempted to measure the far-infrared absorption of $\mathrm{OD}^{-}$impurities in $\mathrm{NaCl}$, in hope that the isotope shift would give more information about the nature of the energy levels. However, we were not able to observe any absorption which could be attributed to $\mathrm{OD}^{-}$impurities. One sample, containing $100 \mathrm{ppm}$ total $\mathrm{OH}^{-}+\mathrm{OD}^{-}$impurities, ${ }^{46}$ gave a spectrum essentially identical with that in Fig. 17, with an absorption strength corresponding to approximately 20-ppm $\mathrm{OH}^{-}$. Wedding and $\mathrm{Klien}^{30}$ were unable to observe the $\mathrm{OD}^{-}$stretching mode in crystals with $\mathrm{OD}^{-}$concentrations estimated in excess of $200 \mathrm{ppm}$. However, changes in the thermal conductivity have been observed for $\mathrm{OD}^{-}$in $\mathrm{NaCl},{ }^{47}$ and the $\mathrm{OD}^{-}$stretching mode has been observed in Raman measurements by Fenner. ${ }^{48}$ Thus, the reason for the absence of any $\mathrm{OD}^{-}$-induced absorption is not at this point understood.

\section{Concentration Dependence}

Bosomworth ${ }^{27}$ has also measured the far-infrared absorption spectrum of $\mathrm{NaCl}: \mathrm{OH}^{-}$, but only for a very-high-impurity concentration. His $1.7^{\circ} \mathrm{K}$ data, for an $\mathrm{OH}^{-}$concentration of $1900 \mathrm{ppm}$ shows a broad absorption band peak at $12 \mathrm{~cm}^{-1}$, but little evidence of any sharp structure. This is clearly different from our data in Fig. 17. We attribute the difference to the factor of about 30 in $\mathrm{OH}^{-}$concentration of the two samples. This would be in agreement with the strongly concentration-dependent absorption seen by Wedding and Klein in the near infrared. In addition, we measured one sample with 1500-ppm $\mathrm{OH}^{-},{ }^{49}$ and found an absorption spectrum very similar to that reported by Bosomworth.

For one crystal, obtained from Harshaw, ${ }^{50}$ with an estimated $\mathrm{OH}^{-}$concentration of $5 \mathrm{ppm}$, we found an absorption spectrum essentially the same as for our 60-ppm samples, except that some of the absorption lines were narrower. This broadening of the absorption lines is also probably due to the $\mathrm{OH}^{-}$concentration difference as opposed to the presence of other impurities. The major unwanted impurity in our samples was $\mathrm{Ca}$. The $\mathrm{Ca}$ concentrations were 0.009 and $0.008 \%$ by weight

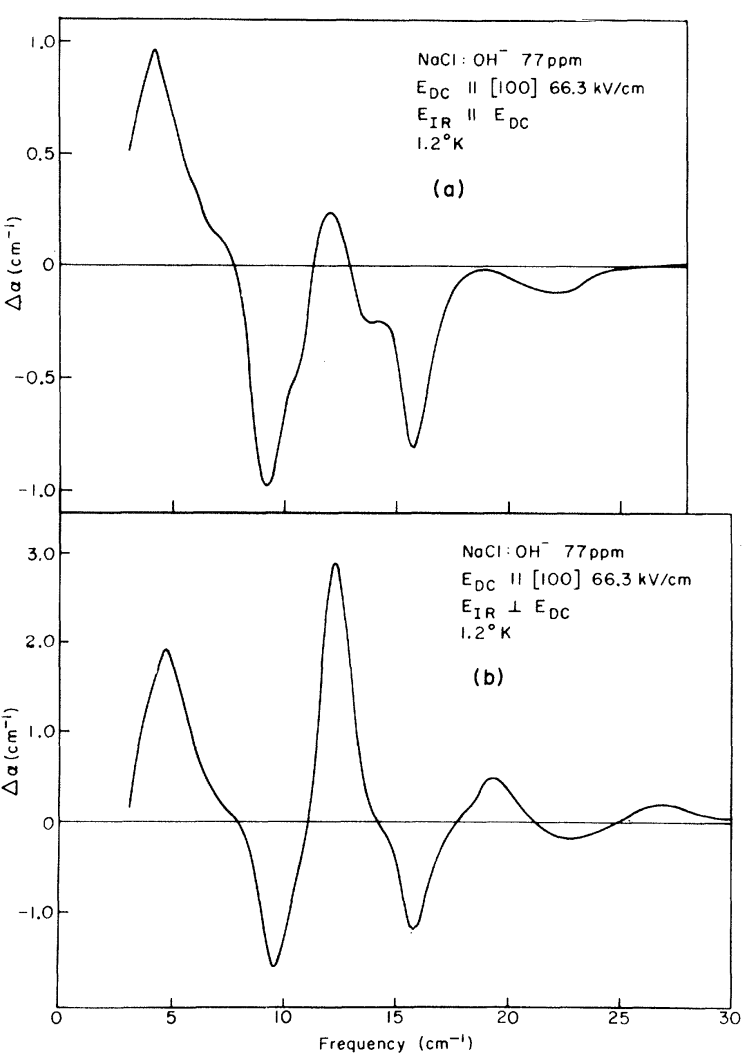

FIG. 19. (a) [100] electric field dependence of the $\mathrm{NaCl}: \mathrm{OH}^{-}$absorption spectrum: $E_{\mathrm{IR}} \| E_{\mathrm{dc}}$. (b) [100] electric field dependence of the $\mathrm{NaCl}: \mathrm{OH}^{-}$absorption spectrum: $E_{\mathrm{IR}} \perp E_{\mathrm{dc}}$.

for the 60-ppm and Harshaw samples, respectively. ${ }^{51}$ The similarity of these Ca concentrations indicates that the broadening of the absorption lines is not due to $\mathrm{Ca}$.

\section{Electric Field Dependence}

Figure 19 (a) shows the electric-field-induced absorption for an external [100] electric field with the $E$ vector of the incident radiation polarized parallel to the applied field. The data shown here were obtained by dividing the field-on transmission spectrum by the field-off transmission spectrum. This technique gives rise to an "electric-field-induced absorption coefficient" $(\Delta \alpha)$. In this case, a negative $\Delta \alpha$ indicates a decrease in absorption with applied field. A positive $\Delta \alpha$ indicates a field-induced increase in absorption at that particular frequency. Clearly, a shift in the absorption frequency of a line will give rise to a negative $\Delta \alpha$ near the unperturbed absorption frequency and a positive $\Delta \alpha$ near the perturbed absorption.

Besides shifts of absorption lines, there are two other effects which are expected to occur for a tunneling system. Some of the transition prob- 
abilities are expected to change as the field is applied. Some transitions which are allowed in zero field will become weaker, and some transitions which are forbidden in zero field will become allowed. In addition, the populations of the tunneling levels are field dependent, so that the absorption strengths will also be field dependent for this reason. In practice, the observed electric-fieldinduced changes in the absorption spectrum will arise from some combination of these three effects.

The first point to notice about Fig. 19 (a) is that $\Delta \alpha$ is negative over most of the frequency range shown. The only regions of positive $\Delta \alpha$ are below $\sim 7 \mathrm{~cm}^{-1}$, and near $12 \mathrm{~cm}^{-1}$. The low-frequency absorption is due to a low-lying energy level shifting upwards with field. The small peak in $\Delta \alpha$ near $12 \mathrm{~cm}^{-1}$ may be due to leakage of radiation polarized perpendicular to the dc field [see Fig. 19 (b) ]. Over all, the remaining part of the spectrum is negative. As can be seen, there are decreases in absorption at $9.3,10.2,13.4^{-1}, 15.7^{-1}$, and $22 \mathrm{~cm}^{-1}$. These effects must be attributed to some combination of decreasing transition probabilities for the transitions involved, and changes in the populations of the initial states for the transitions because of the field-induced splittings of the low-lying levels. It is important to note that the field-induced decrease in absorption at 13.4 $\mathrm{cm}^{-1}$ indicates the presence of a weak absorption line at this frequency in zero field.

Figure 19 (b) shows $\Delta \alpha$ for a [100] electric field and the incident radiation polarized perpendicular to the applied field. The dominant feature here is the strong increase in absorption near $12 \mathrm{~cm}^{-1}$. This absorption line shifts up in frequency with increasing field. Since this line is considerably stronger than the nearby decrease in absorption at $9.6 \mathrm{~cm}^{-1}$, it is not a shift of an existing absorption line. It must be an initially electric-dipoleforbidden transition being made optically active by the electric field.

The absorption peak near $5 \mathrm{~cm}^{-1}$ indicates that a low-lying level is shifting upwards with field. The peak near $18 \mathrm{~cm}^{-1}$, together with the decrease in absorption at $15.7 \mathrm{~cm}^{-1}$, is due to a shift to higher frequencies of the $15.7-\mathrm{cm}^{-1}$ line. Similarly, the $22-\mathrm{cm}^{-1}$ line shifts upwards in frequency with increasing field.

There is a field-induced decrease in absorption at $9.6 \mathrm{~cm}^{-1}$. This is attributed to decreases in the absorption strengths of the $9.3-$ and $10.2-\mathrm{cm}^{-1}$ absorption lines.

At this point, it is clear that the far-infrared absorption spectrum of $\mathrm{NaCl}: \mathrm{OH}^{-}$bears some qualitative resemblence to that of $\mathrm{KCl}: \mathrm{Li}^{+}$. Both systems have low-lying energy levels which are strongly dependent on an applied electric field, and the higher-energy impurity states in both cases show a rapid temperature dependence in the low-temperature region. As will be shown in Sec. $\mathrm{V} \mathrm{B}$ analysis similar to that performed on $\mathrm{KCl}$ : $\mathrm{Li}^{+}$explains many features of the $\mathrm{NaCl}: \mathrm{OH}^{-} \mathrm{ab}-$ sorption spectrum.

\section{B. Formulation of Model}

To explain the complicated absorption spectrum of $\mathrm{NaCl}: \mathrm{OH}^{-}$, and its temperature dependence, we must have mechanisms for producing energy levels within about $2 \mathrm{~cm}^{-1}$ of the ground-state level, as well as 10 to $25 \mathrm{~cm}^{-1}$ above the groundstate level. The purpose of this section is first to consider what types of motional energy states the $\mathrm{OH}^{-}$impurity can have, and then to decide which of the possibilities are appropriate for $\mathrm{OH}^{-}$in $\mathrm{NaCl}$. It will be seen that a model similar to the GBK model can explain many features of the $\mathrm{NaCl}$ : $\mathrm{OH}^{-}$absorption spectrum.

Wedding and Klein ${ }^{30}$ have measured the $\mathrm{OH}^{-}$-induced absorption in $\mathrm{NaCl}$ near the $\mathrm{OH}^{-}$stretching mode at $3650 \mathrm{~cm}^{-1}$. They observed several absorption lines within $20 \mathrm{~cm}^{-1}$ of the stretching mode which presumably correspond in some way to our observed absorption spectrum of Fig. 17. They also found an absorption band $385 \mathrm{~cm}^{-1}$ higher in frequency than the stretching mode, which was attributed to a transition from the first Devonshire manifold ${ }^{52}$ to the second.

Assuming potential minima in the [100] directions for the $\mathrm{O}-\mathrm{H}$ axis orientation, we may use Wedding and Klein's value of $385 \mathrm{~cm}^{-1}$ for the separation of the first and second Devonshire manifolds and use Sauer's tables ${ }^{52}$ to calculate the splittings of the levels in the ground manifold. We find three energy levels: a singlet $A_{1 g}$, a triplet $T_{1 u}$, about $3 \mathrm{~cm}^{-1}$ above the $A_{1 g}$, and a doublet $E_{g}$, about $4.5 \mathrm{~cm}^{-1}$ above the $A_{1 g}$. Such small splittings indicate that there is a barrier which hinders rotation of the $\mathrm{OH}^{-}$molecule of approximately 700 $\mathrm{cm}^{-1}$. Thus, it appears that the Devonshire model can produce the low-lying energy levels necessary to explain the rapid temperature dependence of the $\mathrm{NaCl}: \mathrm{OH}^{-}$absorption spectrum. However, it cannot explain the energy levels in the 10- to 25$\mathrm{cm}^{-1}$ frequency region. A different mechanism is required to produce these levels.

For $\mathrm{KCl}: \mathrm{OH}^{-}$, absorptions at about 0.4 and $0.6 \mathrm{~cm}^{-1}$ have been observed with paraelectric resonance, and a $32-\mathrm{cm}^{-1}$ absorption line has been observed in both near- and far-infrared absorption measurements. ${ }^{27,30,53}$ It should be noted that the Devonshire model predicts "tunneling" splittings of 9 and $14 \mathrm{~cm}^{-1} .{ }^{53}$ The $32-\mathrm{cm}^{-1}$ line shifts down in frequency by a factor of about $1 / \sqrt{2}$ for $\mathrm{OD}^{-} \mathrm{im}$ - 
purities. ${ }^{54}$ To explain these results, Baur and Salzman ${ }^{55}$ proposed that the $\mathrm{OH}^{-}$impurity ion may reside in the $\mathrm{KCl}$ lattice with its molecular c. m. off-center in the [100] direction, and its molecular axis oriented in another, say the [011], direction. Then the $0.4-$ and $0.6-\mathrm{cm}^{-1}$ absorptions could arise because of tunneling of the c. $\mathrm{m}$. between its equilibrium positions, according to the GBK model. The $32-\mathrm{cm}^{-1}$ line would then be due to a transition between librational states. For $\mathrm{OH}^{-}$in $\mathrm{NaCl}$, no isotope-shift data are available, but the rather large tunneling splitting $\left(\sim 2 \mathrm{~cm}^{-1}\right)$ and the reasonable agreement with the Devonshire model indicate that the tunneling may be primarily librational in character.

More recently, Scott and Flygare ${ }^{56}$ have made microwave-absorption measurements in $\mathrm{OH}^{-}-$ doped $\mathrm{KCl}$ and $\mathrm{NaCl}$. Using an extremely sensitive Stark-modulation technique, they observed seven absorption lines between 8.35 and $26.5 \mathrm{GHz}$ in $\mathrm{KCl}: \mathrm{OH}^{-}$. By first adding a strong interaction $\left(V_{6}\right.$ potential) between the $\mathrm{OH}^{-}$ions and the nextnearest-neighbor $\mathrm{Cl}^{-}$ions to the Devonshire mod$\mathrm{el}$, and then taking into account the quasistatic $C_{4 v}$ symmetry potential which would be present if the center of rotation of the $\mathrm{OH}^{-}$ion were not at the host-ion lattice site, Scott and Flygare were able to fit their observed absorption frequencies, as well as explain the available paraelectric-resonance results. It is significant that their model is also able to explain the observed $32-\mathrm{cm}^{-1}$ band in $\mathrm{KCl}: \mathrm{OH}^{-}$, since the strong $V_{6}$ potential brings the Devonshire librator levels and the low-lying tunneling levels closer together.

One result of Scott and Flygare's measurements is very difficult to understand. The absorption linewidths they observed were typically less than a few hundred $\mathrm{MHz}$. For the impurity concentrations used ( $1 \mathrm{ppm})$, dipole-dipole broadening and strain broadening due to the $\mathrm{OH}^{-}$ions themselves give linewidths of the same order as those observed. At such low-impurity concentrations, one would expect dislocation broadening to be dominant anyway, giving rise to strains of $10^{-4}$ or $10^{-5}$ in the host lattice. For example, in high-purity samples of $\mathrm{KCl}$ : $\mathrm{OH}^{-}$, Bron and Dreyfus ${ }^{13}$ found a dislocation count of $2 \times 10^{6}$ dislocation lines $/ \mathrm{cm}^{2}$, which corresponds to inhomogeneous strains of $2 \times 10^{-5}$. Strains of this magnitude indicate a linewidth of $0.1-0.2 \mathrm{~cm}^{-1} 5 \mathrm{GHz}$ for the $\mathrm{KCl}: \mathrm{OH}^{-}$ paraelectric-resonance transitions. ${ }^{13}$ This calculated broadening is more than an order of magnitude larger than the linewidths observed by Scott and Flygare.

In $\mathrm{NaCl}: \mathrm{OH}^{-}$, Scott and Flygare found only two absorption lines, at 0.6 and $0.9 \mathrm{~cm}^{-1}$. There may have been other lines outside the range of their microwave spectrometer $\left(0.27\right.$ to $\left.1.3 \mathrm{~cm}^{-1}\right)$. They attempted to fit the model to their observed lines and our far-infrared results, with only limited success. While their model can explain the presence of both low-lying tunneling levels as well as energy levels in the $10-$ to $25-\mathrm{cm}^{-1}$ region, it predicts some strong lines which are not observed (at 4 and $26 \mathrm{~cm}^{-1}$ ), and assigns some of the observed strong lines (at 9.3 and $15.7 \mathrm{~cm}^{-1}$ ) to transitions which should be weak. In addition, the model fails to quantitatively explain the observed temperature dependence of the $9.3-$ and $15.7-\mathrm{cm}^{-1}$ lines. Thus, while their model appears to explain the available data on $\mathrm{KCl}: \mathrm{OH}^{-}$, it fails to explain the far-infrared results on $\mathrm{NaCl}: \mathrm{OH}^{-}$.

In view of the preceding results and discussion, we will take a different approach in the interpretation of our experimental results on $\mathrm{NaCl}: \mathrm{OH}^{-}$. It is worth pointing out here that there are two main differences between our model to be described and that of Scott and Flygare. Whereas they assume that the c. m. of the $\mathrm{OH}^{-}$ion is essentially "frozen" off-center in a [100] direction and the molecule therefore rotates in a $C_{4 v}$ potential, we assume that there is effectively a correlation between any off-center displacement of the c. $m$. and the direction of the molecular axis. In other words, if the molecule "rotates" or tunnels through $90^{\circ}$, the c. m. displacement also rotates through $90^{\circ}$. Second, we obtain energy levels in the region $10-25 \mathrm{~cm}^{-1}$ by introducing "translational" motion of the whole molecule, i. e., a low-frequency vibrational mode of the c.m. Scott and Flygare achieve levels in the same region by introducing the $V_{6}$ potential to bring down higher rotational states, followed by the splitting due to the $C_{4 v}$ symmetry of their "hindered rotator."

We first assume that the $\mathrm{OH}^{-}$molecule substitutes for a $\mathrm{Cl}^{-}$ion in the $\mathrm{NaCl}$ lattice. Bron and Dreyfus $^{13}$ have pointed out that because of the large discrepancy between the dipole moment of $\mathrm{OH}^{-}$in $\mathrm{KCl}$ as determined from paraelectric resonance and dielectric measurements as compared with that estimated from near-infrared measurements, it is likely that the $\mathrm{OH}^{-}$ion occupies a [100] off-center position. We also make this assumption for $\mathrm{OH}^{-}$in $\mathrm{NaCl}$, although it will make no qualitative difference in the results of the calculation.

In Fig. 20 (a) are shown the six equivalent [100] equilibrium position orientations for the $\mathrm{OH}^{-}$ molecule. We first want to consider the motion of the impurity when it is frozen in a particular equilibrium site, say $a$. As for $\mathrm{KCl}: \mathrm{Li}^{+}$, this involves choosing a potential-energy function $V_{a}$ $(x, y, z)$ which closely approximates the real local well, but which does not permit the impurity to 


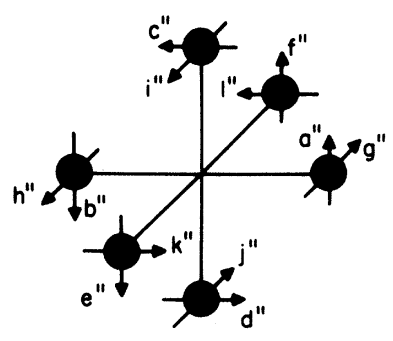

E - Excited State Bosis Stotes

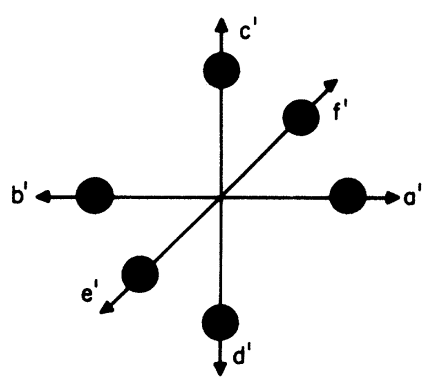

$A_{1}$ - Excited Stote Bosis Stotes
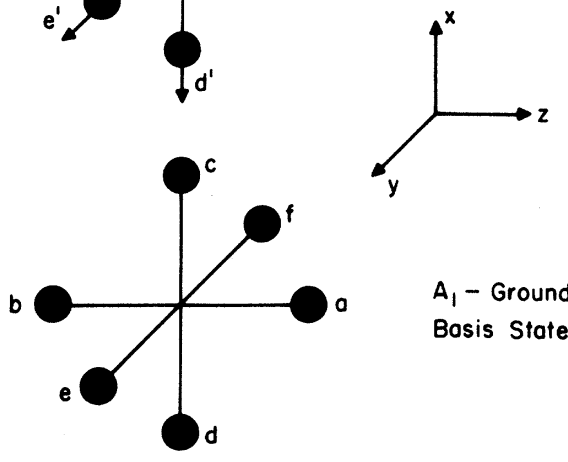

$A_{1}$ - Ground State Bosis States

FIG. 20. (a) $\mathrm{NaCl}$ : $\mathrm{OH}^{-}$tunneling-model ground-state basis states. (b) $\mathrm{NaCl}$ : $\mathrm{OH}^{-}$tunneling-model basis states for the $A_{1}$-symmetry resonant-mode excited state. (c) $\mathrm{NaCl}$ : $\mathrm{OH}^{-}$tunneling-model basis states for the $E$-symmetry resonant-mode excited state.

leave the vicinity of site $a$. Then, neglecting the $\mathrm{O}-\mathrm{H}$ stretching vibrations, the approximate Hamiltonian $H_{o}$ for the motion in site $a$ may be written

$$
H_{o}=H_{T}+H_{L} \text {, }
$$

where $H_{T}$ is the Hamiltonian governing the c. m. motion, and $H_{L}$ determines the motion of the molecule about its c. m. Since no coupling between the two (orthogonal) types of motion has been introduced, the wave functions representing the motion of the $\mathrm{OH}^{-}$molecule will then be a product of the translational motion wave functions $\phi_{T}^{n}$, and the librational motion wave functions $\phi_{L}^{m}$ so

$$
\psi_{n m}=\phi_{T}^{n} \phi_{L}^{m}, \quad m, n=0,1,2, \ldots .
$$

From near-infrared measurements on this system, it appears that the first excited state for librational motion is $385 \mathrm{~cm}^{-1}$ above the (product) ground state. Thus, in the far-infrared spectral region, we need only consider the librator ground state. Hence, the ground and first excited states for the motion of the $\mathrm{OH}^{-}$molecule when it is fixed in this one position are

$$
\begin{array}{ll}
\psi_{o o}=\phi_{T}^{o} \phi_{L}^{o} & \text { (ground state) }, \\
\psi_{1 o}=\phi_{T}^{1} \phi_{L}^{o} & \text { (first excited state). }
\end{array}
$$

In this picture, the translational motion of the c. $m$. system occurs in a local potential well of $C_{4 v}$ symmetry, so that the first excited state for this motion will consist of two levels, of $A_{1}$ and $E$ symmetries. The $A_{1}$ level corresponds to translational motion parallel to the $C_{4}$ axis (and thus parallel to the molecular axis), and the twofold degenerate $E$ level involves motion in the plane perpendicular to the $C_{4}$ axis.

There will also be other such states, corresponding to localization of the $\mathrm{OH}^{-}$molecule in each of the five remaining equilibrium sites. Referring to Fig. 20 (a), the states $|a\rangle,|b\rangle, \ldots,|f\rangle$ represent the approximate ground-state translation-libration product wave functions. However, the $\mathrm{OH}^{-}$molecule cannot be considered to be completely localized in one [100] position. There are large, but finite, potential barriers hindering the molecule from moving from one equilibrium position to another, and the zero-point translationallibrational motion will induce the $\mathrm{OH}^{-}$molecule to tunnel between them.

This tunneling of the $\mathrm{OH}^{-}$ion between the equilibrium positions will be described by the matrix elements of the real multiwell Hamiltonian $H$ connecting the equilibrium states $|a\rangle, \ldots,|f\rangle$. There will, for example, be matrix elements of the form $\langle a|H| c\rangle$ connecting the ground-state basis states $|a\rangle$ and $|c\rangle$.

We assume that

$$
\langle a|H| b\rangle=\langle c|H| d\rangle=\langle e|H| f\rangle=0,
$$

so that only $90^{\circ}$ tunneling is allowed. This allows us to write down a matrix Hamiltonian in terms of the matrix elements:

$$
\text { - } \Delta=\langle a|H| c\rangle=\langle a|H| d\rangle=\langle a|H| e\rangle=\cdots \text {. }
$$

This problem has been formally solved by Shore, ${ }^{4}$ and independently be Sauer, Schirmer, and Schneider, ${ }^{5}$ so we will only quote their results. They found that the ground state is split into three levels with energies $-2 \Delta, 0$, and $+\Delta$, and symmetries $A_{1 g}, T_{1 u}$, and $E_{g}$, respectively. We note here that this result is qualitatively the same as the splitting of the Devonshire ground manifold. We attribute the observed $2-\mathrm{cm}^{-1}$ absorption to the $A_{1 g} \rightarrow T_{1 u}$ transition, and hence take $\Delta=1 \mathrm{~cm}^{-1}$. This compares favorably with our earlier estimate of $\Delta=1.5 \mathrm{~cm}^{-1}$ on the basis of the Devonshire mod$\mathrm{el}$, and thus indicates that the tunneling rates may be primarily determined by the librational character of the motion. 
As pointed out earlier, the first-excited-state motion for the $\mathrm{OH}^{-}$impurity in a particular equilibrium site will consist of two levels, corresponding to the $A_{1}$ and $E$ irreducible representations of the $C_{4 v}$ symmetry group. The basis states for the $A_{1}$-symmetry excited-state level are shown schematically in Fig. 20 (b). Here, the arrows indicate that a resonant-mode quantum corresponding to translational motion parallel to the molecular axis has been excited. Because the symmetry is the same, this level will be tunnel split in the same manner as the ground state.

Thus if we define

$$
-\Delta^{\prime}=\left\langle a^{\prime}|H| c^{\prime}\right\rangle=\cdots,
$$

and ignore $180^{\circ}$ tunneling, we again find three energy levels, at $E_{0}^{\prime}-2 \Delta^{\prime}$, and $E_{0}^{\prime}$, and $E_{0}^{\prime}+\Delta^{\prime}$, with symmetries $A_{1 g}, T_{1 u}$, and $E_{g}$, respectively. Here $E_{0}^{\prime}$ is the energy of the $A_{1}$-symmetry resonantmode level before the tunneling splitting. To fit the experimental results, we take $\Delta^{\prime}=\Delta=1 \mathrm{~cm}^{-1}$. Then the lowest-frequency electric-dipole-allowed transition is $T_{1 u}$ (ground) $\rightarrow A_{1 g}$ (excited). We assign this transition to the observed absorption line at $9.3 \mathrm{~cm}^{-1}$ (i. e., $E_{0}^{\prime}=11.3 \mathrm{~cm}^{-1}$ ). The other electric-dipole-allowed transition energies are then $10.3,12.3$, and $13.3 \mathrm{~cm}^{-1}$.

The tunneling splitting of the $E$-symmetry resonant-mode excited state is calculated in a similar manner. We start with the twelve-fold-degenerate basis states shown schematically in Fig. 20 (c). The balls indicate the $\mathrm{OH}^{-}$orientation, and the arrows indicate which of the two possible resonant-mode quanta has been singly excited. From group-theoretic considerations, there are four triply degenerate energy levels, corresponding to the irreducible representations $T_{1 u}, T_{2 u}$, $T_{1 g}$, and $T_{2 g}$ of the $O_{h}$ symmetry group. In order to write down a matrix Hamiltonian, we make the same assumption as was made in the calculation of the ground-state splitting, i. e., only $90^{\circ}$ tunneling is important. Then there are only two different types of Hamiltonian matrix elements: those of the form

TABLE II. E-symmetry excited-state tunneling levels in terms of linear combinations of the resonant-mode excited-state basis states.

\begin{tabular}{cc}
\hline \hline$T_{1 u}\left(E=E_{0}^{\prime \prime}-2 \beta\right)$ & $T_{1 g}\left(E=E_{0}^{\prime \prime}+2 \alpha\right)$ \\
\hline$\frac{1}{2}\left(a^{\prime \prime}-b^{\prime \prime}+f^{\prime \prime}-e^{\prime \prime}\right)$ & $\frac{1}{2}\left(a^{\prime \prime}+b^{\prime \prime}+c^{\prime \prime}+d^{\prime \prime}\right)$ \\
$\frac{1}{2}\left(c^{\prime \prime}-d^{\prime \prime}+l^{\prime \prime}-k^{\prime \prime}\right)$ & $\frac{1}{2}\left(e^{\prime \prime}+f^{\prime \prime}+i^{\prime \prime}+j^{\prime \prime}\right)$ \\
$\frac{1}{2}\left(g^{\prime \prime}-h^{\prime \prime}+j^{\prime \prime}-i^{\prime \prime}\right)$ & $\frac{1}{2}\left(g^{\prime \prime}+h^{\prime \prime}+l^{\prime \prime}+k^{\prime \prime}\right)$ \\
$T_{2 u}\left(E=E_{0}^{\prime \prime}+2 \beta\right)$ & $T_{2 g}\left(E=E_{0}^{\prime \prime}-2 \alpha\right)$ \\
$\frac{1}{2}\left(a^{\prime \prime}-b^{\prime \prime}+e^{\prime \prime}-f^{\prime \prime}\right)$ & $\frac{1}{2}\left(a^{\prime \prime}+b^{\prime \prime}-c^{\prime \prime}-d^{\prime \prime}\right)$ \\
$\frac{1}{2}\left(c^{\prime \prime}-d^{\prime \prime}+l^{\prime \prime}-k^{\prime \prime}\right)$ & $\frac{1}{2}\left(e^{\prime \prime}+f^{\prime \prime}-i^{\prime \prime}-j^{\prime \prime}\right)$ \\
$\frac{1}{2}\left(g^{\prime \prime}-h^{\prime \prime}+i^{\prime \prime}-j^{\prime \prime}\right)$ & $\frac{1}{2}\left(g^{\prime \prime}+h^{\prime \prime}-k^{\prime \prime}-l^{\prime \prime}\right)$ \\
\hline
\end{tabular}

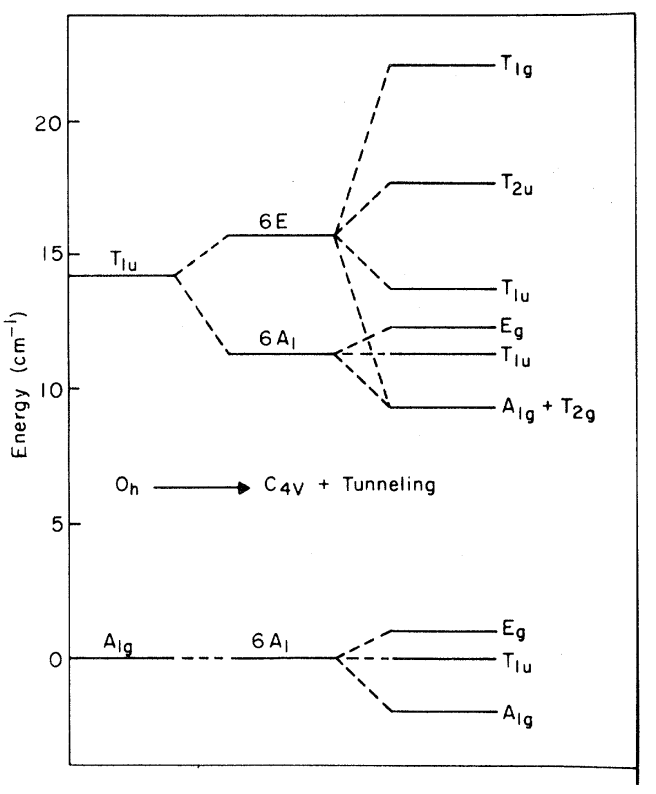

FIG. 21. Development of the NaCl: $\mathrm{OH}^{-}$tunnelingmodel energy-level scheme.

$$
\alpha=\left\langle a^{\prime \prime}|H| c^{\prime \prime}\right\rangle
$$

and those like

$$
-\beta=\left\langle a^{\prime \prime}|H| f^{\prime \prime}\right\rangle \text {. }
$$

The linear combinations of the basis functions that diagonalize the resulting matrix Hamiltonian are listed in Table II. The eigenvalues for these levels are

$$
\begin{array}{ll}
E\left(T_{1 u}\right)=E_{0}^{\prime \prime}-2 \beta, & E\left(T_{2 u}\right)=E_{0}^{\prime \prime}+2 \beta, \\
E\left(T_{1 g}\right)=E_{0}^{\prime \prime}+2 \alpha, & \text { and } E\left(T_{2 g}\right)=E_{0}^{\prime \prime}-2 \alpha .
\end{array}
$$

Here $E_{0}^{\prime \prime}$ is the energy of the $E$-symmetry resonant-mode level before the tunneling splitting. Now all that remains is to specify the splitting parameters $\alpha$ and $\beta$, the $A_{1}-E$ resonant-mode excited-state splitting, and the energy levels resulting from this model will be determined.

Because of its temperature dependence, the $15.7-\mathrm{cm}^{-1}$ absorption line must arise from a transition for which the initial state is the $A_{1 g}$ level in the ground-state manifold. By symmetry, the final state for this transition must be the $T_{1 u}$ level of the $E$-symmetry excited-state manifold. So we fix this $T_{1 u}$ level to be $15.7 \mathrm{~cm}^{-1}$ higher in energy than the $A_{1 g}$ ground state. Because of its temperature dependence, we assign the observed $22-\mathrm{cm}^{-1}$ line to the $T_{1 u}$ (ground) to $T_{2 g}$ transition. This leaves only one parameter unspecified, namely, the resonant-mode excited-state $A_{1}-E$ splittings. To fit the observed absorption frequencies, we choose this to be $4.4 \mathrm{~cm}^{-1}$, so that $E_{0}^{\prime \prime}=17.7 \mathrm{~cm}^{-1}$. 
The development of these energy levels is shown in Fig. 21.

We may also calculate transition probabilities for these transitions. The dipole matrix connecting the ground-state basis states with the $A_{1}$-symmetry excited basis states is

$$
\mu^{\prime}\left[\begin{array}{cccccc}
\hat{k} & 0 & 0 & 0 & 0 & 0 \\
0 & -\hat{k} & 0 & 0 & 0 & 0 \\
0 & 0 & \hat{i} & 0 & 0 & 0 \\
0 & 0 & 0 & -\hat{i} & 0 & 0 \\
0 & 0 & 0 & 0 & \hat{j} & 0 \\
0 & 0 & 0 & 0 & 0-\hat{j}
\end{array}\right]
$$

where $\hat{i}, \hat{j}$, and $\hat{k}$ are unit vectors in the $x, y, z$ [100] directions, respectively. Here,

$$
\vec{\mu}^{\prime}=\left|\vec{\mu}_{a}^{\prime}\right|=\left\langle a\left|e^{* \overrightarrow{\mathrm{r}}}\right| a^{\prime}\right\rangle=\left\langle b\left|e^{* \overrightarrow{\mathrm{r}}}\right| b^{\prime}\right\rangle=\cdots
$$

is the transition dipole matrix element connecting basis state $\left|a^{\prime}\right\rangle$ with state $\left|b^{\prime}\right\rangle$ with $|b\rangle$, etc., and $e^{*}$ is the effective charge associated with the $\mathrm{OH}^{-}$ion.

The dipole matrix connecting the ground-state basis states with the $E$-excited basis states is

$$
\mu^{\prime \prime}\left[\begin{array}{rrrrrrrrrrrr}
\hat{i} & 0 & 0 & 0 & 0 & 0 & -\hat{j} & 0 & 0 & 0 & 0 & 0 \\
0 & -\hat{i} & 0 & 0 & 0 & 0 & 0 & \hat{j} & 0 & 0 & 0 & 0 \\
0 & 0 & -\hat{k} & 0 & 0 & 0 & 0 & 0 & \hat{j} & 0 & 0 & 0 \\
0 & 0 & 0 & \hat{k} & 0 & 0 & 0 & 0 & 0 & -\hat{j} & 0 & 0 \\
0 & 0 & 0 & 0 & -\hat{i} & 0 & 0 & 0 & 0 & 0 & \hat{k} & 0 \\
0 & 0 & 0 & 0 & 0 & \hat{i} & 0 & 0 & 0 & 0 & 0 & -\hat{k}
\end{array}\right],
$$

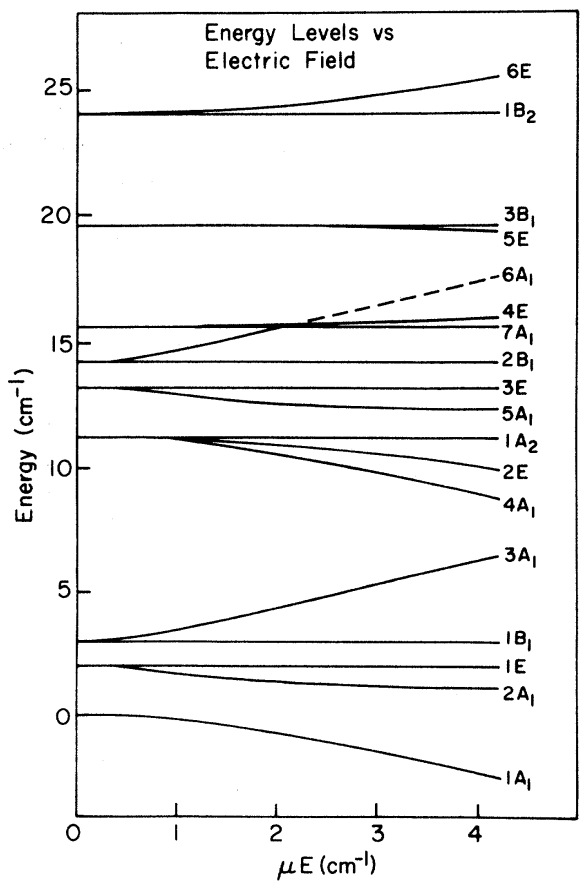

FIG. 22. [100] electric field dependence of the model energy levels.

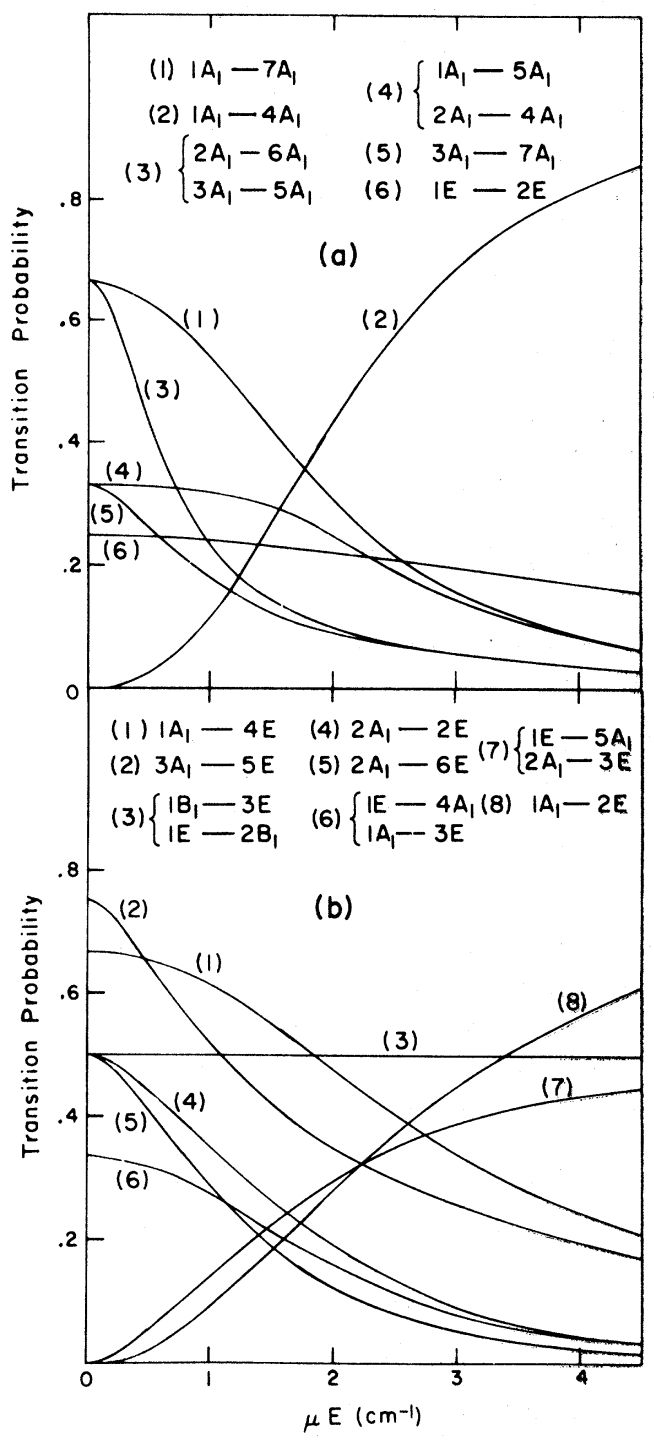

FIG. 23. (a) Calculated transition probabilities for a [100] electric field and $E_{\mathrm{IR}} \| E_{\mathrm{dc}}$. (b) Calculated transition probabilities for a [100] electric field and $E_{1 R} \perp E_{\mathrm{dc}}$.

where $\mu^{\prime \prime}$ is the dipole moment for transitions between the ground-state basis states and the $E$ symmetry basis states. We note that $\mu^{\prime}$ and $\mu^{\prime \prime}$ are not, in general, of equal magnitude.

The effect of applying an external electric field can also be included in this calculation. This involves adding the dipolar energies to the appropriate matrix elements in the three Hamiltonian matrices we have used. This calculation has been done previously for the ground-state Hamiltonian matrix, and thus also for the $A_{1}$ excited-state Hamiltonian matrix. The extension to the $E$ excited-state manifold is straightforward. We have solved the three resulting secular equations for a [100] electric field. The splittings of the energy 
TABLE III. Comparison of tunneling model with observed $\mathrm{NaCl}$ : $\mathrm{NaOH}$.

\begin{tabular}{lccc}
\hline \hline $\begin{array}{c}\text { Allowed } \\
\text { transitions }\end{array}$ & $\begin{array}{c}\text { Absorption spectrum } \\
\text { Predicted } \\
\text { frequency } \\
\left(\mathrm{cm}^{-1}\right)\end{array}$ & $\begin{array}{c}\text { Observed } \\
\text { frequency } \\
\left(\mathrm{cm}^{-1}\right)\end{array}$ & $\begin{array}{c}\text { Predicted } \\
\text { transition } \\
\text { probability }\end{array}$ \\
\hline$T_{1 u}(G) \rightarrow E_{g}(G)^{\mathrm{a}}$ & 1.0 & n.o. & $\frac{2}{3} \mu^{2}$ \\
$A_{1 g}(G) \rightarrow T_{1 u}(G)$ & 2.0 & 2 & $\frac{1}{3} \mu^{2}$ \\
$T_{1 u}(G) \rightarrow A_{1 g}\left(A_{1}\right) \mathrm{c}$ & 9.3 & 9.3 & $\frac{1}{3} \mu^{2}$ \\
$T_{1 u}(G) \rightarrow T_{2 g}(E)^{\mathrm{c}}$ & 9.3 & 9.3 & $\mu^{2}$ \\
$E_{\boldsymbol{g}}(G) \rightarrow T_{1 u}\left(A_{1}\right)$ & 10.3 & 10.2 & $\frac{2}{3} \mu^{2}$ \\
$T_{1 u}(G) \rightarrow E_{g}\left(A_{1}\right)$ & 12.3 & 12.2 & $\frac{2}{3} \mu^{2}$ \\
$E_{g}(G) \rightarrow T_{1 u}(E)$ & 12.7 & $(?)$ & $\frac{1}{3} \mu^{2}$ \\
$A_{1 g}(G) \rightarrow T_{1 u}\left(A_{1}\right)$ & 13.3 & $\sim 13.4^{\mathrm{d}}$ & $\frac{1}{3} \mu^{2}$ \\
$A_{1 g}(G) \rightarrow T_{1 u}(E)$ & 15.7 & 15.7 & $\frac{2}{3} \mu^{2}$ \\
$E_{\boldsymbol{g}}(G) \rightarrow T_{2 u}(E)$ & 16.7 & $\mathrm{n} .0$. & $\mu^{2}$ \\
$T_{1 u}(G) \rightarrow T_{1 g}(E)$ & 22.1 & 22 & $\mu^{2}$ \\
\hline \hline
\end{tabular}

${ }^{\mathrm{a}} G$ indicates ground-state tunneling level.

$b_{n}$.o. indicates that the absorption line was not observed.

${ }^{\mathrm{c}} A_{1}$ indicates $A_{1}$ excited-state tunneling level and $E$ indicates $E$ excited-state tunneling level.

Oobserved as a decrease in absorption with an applied electric field.

levels as a function of field for our particular choice of tunneling parameters are shown in Fig. 22. Figures 23 (a) and 23 (b) show the transition probabilities as a function of electric field for some of the stronger transitions. It should be pointed out that for the purpose of these numerical calculations, we have assumed that

$$
\begin{gathered}
\left\langle a\left|e^{* \overrightarrow{\mathrm{r}}}\right| a\right\rangle=\left\langle a^{\prime}\left|e^{* \overrightarrow{\mathrm{r}}}\right| a^{\prime}\right\rangle=\left\langle a^{\prime \prime}\left|e^{* \overrightarrow{\mathrm{r}}}\right| a^{\prime \prime}\right\rangle . \\
\text { C. Comparison of Model with Experiment }
\end{gathered}
$$

\section{Temperature Dependence}

In Table III is shown a comparison between the absorption frequencies predicted by the model and those actually observed. The model uses six parameters $\left(\Delta, \Delta^{\prime}, \alpha, \beta, E_{0}^{\prime}\right.$, and $\left.E_{0}^{\prime \prime}\right)$ to predict the observed absorption lines at $2,9.3,10.2,12.2$, $13.4,15.7$, and $22 \mathrm{~cm}^{-1}$. It does not predict the very weak absorption line observed at $11.1 \mathrm{~cm}^{-1}$. (The model has transitions at $11.3 \mathrm{~cm}^{-1}$ but they are electric dipole forbidden.) No evidence of the predicted $16.7-\mathrm{cm}^{-1}$ line was observed. The model predicts only one other absorption line, at 12.7 $\mathrm{cm}^{-1}$. This line is expected to be weak, and if indeed it is present, it could be buried in the rather large background absorption in this frequency region.

The strengths of the absorption lines are expected to vary rapidly with temperature near $1^{\circ} \mathrm{K}$ because of the rapidly changing relative populations of the levels in the ground-state tunneling multiplet. The relative populations of these levels, as predicted by the model, are shown in Fig. 24. In

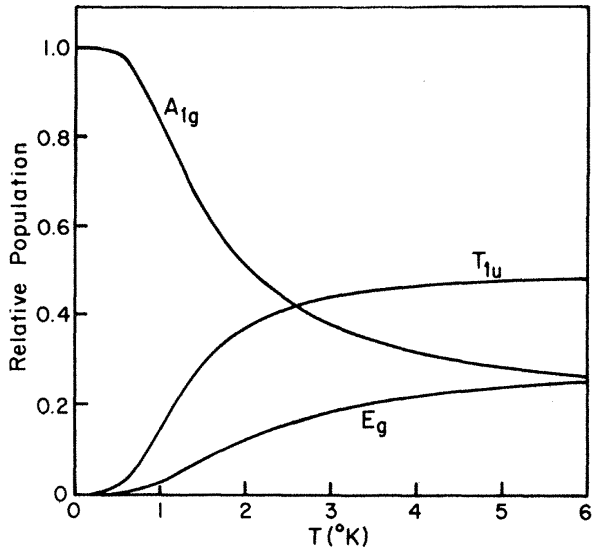

FIG. 24. Boltzmann populations of the model groundstate tunneling levels.

Fig. 25, the observed absorption strengths of the 15.7- and $22-\mathrm{cm}^{-1}$ lines are shown as a function of temperature. The solid lines are the predicted temperature dependences based on the relative populations of the initial state for the transition. Since the observed relative absorption strengths do not agree with the predictions of the model, the theoretical curves have been scaled to give a best fit. The remaining absorption lines are too obscured by adjacent absorptions for such a comparison to be made. However, qualitatively the 9.3-, 10.2-, and $12.2-\mathrm{cm}^{-1}$ absorption lines do increase in strength with increasing temperature, as predicted by the model.

\section{Electric Field Dependence}

As was shown, the model predicts a rather strong electric field dependence for most of the absorption lines. There are two kinds of effects

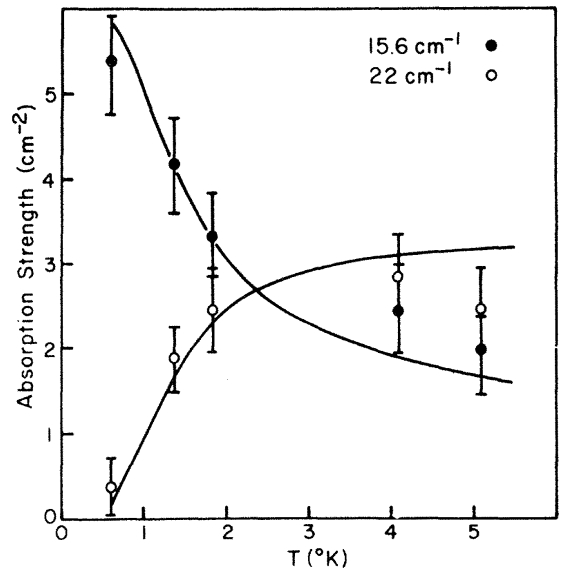

FIG. 25. Temperature dependence of the 15.7- and $22-\mathrm{cm}^{-1}$ absorption line strengths. The solid curves are the temperature dependences calculated from the model. 
which can occur, namely, shifts in the absorption frequencies, and changes in the absorption strengths. In reality, the dichroisms do not provide a sensitive qualitative test of the model, since most lines which are allowed in zero field are predicted to become weaker in the presence of a field, and many transitions which are forbidden in zero field are expected to become allowed. Thus, only if a quantitative comparison between experiment and theory can be made are the dichroisms a real test of the model. This type of comparison is not possible in any detail because of the many broad and overlapping absorption lines, and because of the manner in which the electric field data is taken. In view of this, we will limit ourselves to a semiquantitative comparison distinguishing only between dichroisms which are predicted to be large enough to be easily observable or too small to be observable.

On this basis, there is agreement between experiment and theory for all of the observed absorption lines but one. The model predicts an increase in absorption at $11.3 \mathrm{~cm}^{-1}$ for light polar.ized both parallel and perpendicular to the external [100] electric field. Such an absorption was observed only for perpendicular polarization. On the basis of the model, this increase in absorption must come from some combination of the following transitions: $1 A_{1} \rightarrow 2 E, 2 A_{1} \rightarrow 3 E$, and $1 E \rightarrow 5 A_{1}$ (referring to Fig. 22). Of these, the model predicts that the first transition will be the most important. This is consistent with our observation of a weak absorption line near $11 \mathrm{~cm}^{-1}$ at $0.6^{\circ} \mathrm{K}$ with no external electric field.

We can estimate the magnitude of the inhomogeneous electric field at the site of an $\mathrm{OH}^{-}$impurity due to the random distribution of $\mathrm{OH}^{-}$dipoles in the crystal. For this we use the statistical method described by Stoneham. ${ }^{57}$ This method is not suited to a calculation of the mean magnitude $\bar{E}$ of the field, but gives the probability distribution of the $z$ component $E_{z}$ of the field. A calculation shows that this distribution $I(z)$ is a Lorentzian peaked at $E_{z}=0$ with a full half-width of 8.97 $\rho \mu / \epsilon$, where $\rho$ is the density of dipoles of moment $\mu$ and $\epsilon$ is the dielectric constant. This compares with a rough estimate by Bron and Dreyfus which gave $\bar{E}=6 \rho \mu / \epsilon$. If $\mu=0.9 \mathrm{e} \AA$ (see Sec. VI), then we find the full half-width of the $E_{z}$ distribution to be $9 \mathrm{kV} / \mathrm{cm}$ for an $\mathrm{OH}^{-}$concentration of $60 \mathrm{ppm}$. The large electric-field-induced absorption near $11.3 \mathrm{~cm}^{-1}$ suggests that random fields of this order could give rise to the observed weak absorption in zero field.

We note that for $\mathrm{OH}^{-}$concentrations of 1500-2000 $\mathrm{ppm}$, there will be random dipolar electric fields on the order of $200 \mathrm{kV} / \mathrm{cm}$ in the crystal. In view
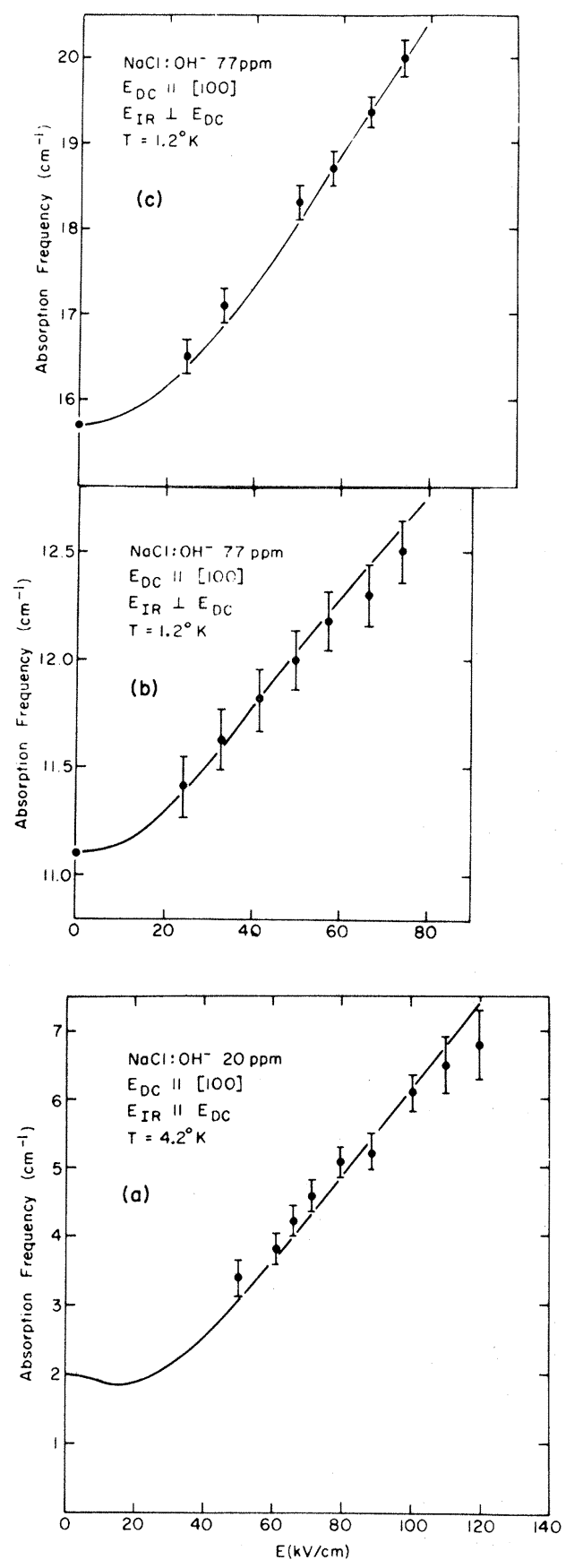

FIG. 26. (a) [100] electric-field-induced frequency shift of a low-lying tunneling level. The solid curve is the calculated field dependence of the $1 A_{1} \rightarrow 2 A_{1}$ transition of Fig. 22 assuming an $\mathrm{OH}^{-}$dipole moment of 0.89 $e \AA$. (b) [100] electric-field-induced frequency shift of the $15.7-\mathrm{cm}^{-1}$ absorption line. The solid curve is the calculated field dependence of the $1 A_{1} \rightarrow 4 E$ transition of Fig. 22 assuming an $\mathrm{OH}^{-}$dipole moment of $0.94 e \AA$. (c) $[100]$ electric-field-induced frequency shift of the $11.1-\mathrm{cm}^{-1}$ absorption line. The solid curve is the calculated field dependence of the $1 A_{1} \rightarrow 2 E$ transition of Fig. 22 assuming an $\mathrm{OH}^{-}$dipole moment of $0.89 e \AA$. 
of the observed electric field results, this could account for a large share of the observed concentration dependence.

Electric-field-induced shifts of some of the absorption lines were also observed. Here again there is some difficulty in making a quantitative comparison between experiment and theory, because not all of the absorption lines are well enough separated to measure the frequency shifts, or in some cases, to even determine if one has occurred.

With this qualification in mind, we now compare experiment and theory for three of the absorption lines. Figure 26 (a) shows the frequency-versusfield curve for a ground-state tunneling transition. The external field was in the [100] direction, and the radiation was polarized parallel to the field. This absorption line is attributed to the $1 A_{1} \rightarrow 2 A_{1}$ transition of Fig. 22. The solid curve used to fit the data is the separation of the $1 A_{1}$ and $2 A_{1}$ levels as a function of field assuming an $\mathrm{OH}^{-}$dipole moment of $0.89 e \AA$.

Figure $26(\mathrm{~b})$ shows the shift of the $11.1-\mathrm{cm}^{-1}$ line for $E_{\mathrm{IR}} \perp E_{\mathrm{dc}}$. The solid curve is that predicted by the model assuming the absorption line arises from the $1 A_{1} \rightarrow 2 E$ transition with an $\mathrm{OH}^{-}$ dipole moment of $0.89 \mathrm{e} \AA$.

Figure 26 (c) shows the shift of the $15.7-\mathrm{cm}^{-1}$ line for $E_{\mathrm{IR}}$ perpendicular to the $[100]$ external field. The solid curve is the predicted field dependence assuming an $\mathrm{OH}^{-}$dipole moment of 0.94 $e \AA$. It should be pointed out that no corresponding shift was observed for $E_{\mathrm{IR}} \| E_{\mathrm{dc}}$ even though it is predicted by the model. Part of the reason for this could lie in the fact that the $15.7-\mathrm{cm}^{-1}$ line decreases in strength with increasing field much more rapidly for $E_{\mathrm{IR}} \| E_{\mathrm{dc}}$ than for $E_{\mathrm{IR}} \perp E_{\mathrm{dc}}$.

Thus, for these three absorption lines, we have found some agreement with the model. The measured dipole moments are the same to within about $10 \%$ for all three lines. The value of $\mu=0.9 e \AA$ compares reasonably well with the value $\mu=0.7 e \AA$ which has been measured for $\mathrm{OH}^{-}$impurities in $\mathrm{KCl},{ }^{14}$ and with the value $\mu=1.0 e \AA$ for $\mathrm{OH}^{-}$in $\mathrm{KBr} .{ }^{12}$

Thus, while the model can explain most features of the $\mathrm{NaCl}: \mathrm{OH}^{-}$absorption spectrum, it does not explain all of the experimental results. Some of its failures can certainly be eliminated by simple modifications and straightforward extensions of the model. However, this approach will not be justified until complementary experimental evidence is obtained which shows that c.m. resonantmode motion is indeed primarily responsible for the $9.3-$ to $22-\mathrm{cm}^{-1}$ absorption lines.

\section{SUMMARY}

For $\mathrm{KCl}: \mathrm{Li}^{+}$, the energy levels in the tunneling multiplet are found to be well described by the model of Gomez et al. for a [111] off-center defect. A dipole moment of $1.14 \pm 0.05 e \AA$ is measured for both $\mathrm{Li}^{6}$ and $\mathrm{Li}^{7}$ impurities. The linewidths of the tunneling transitions indicate the presence of inhomogeneous lattice strains. The $\mathrm{KCl}$ : $\mathrm{Li}^{+} 40-\mathrm{cm}^{-1}$ band shows a rapid temperature dependence in the 1 to $4{ }^{\circ} \mathrm{K}$ temperature region, indicating that it is due to transitions from the tunneling multiplet. The most surprising feature of this band is that it has a negative isotope shift, where the absorption frequency for isotopic $\mathrm{Li}^{6}$ impurities is smaller than that due to $\mathrm{Li}^{7}$ impurities. Very little agreement is found between the experimental results on the $40-\mathrm{cm}^{-1}$ band and an extension of the [111] tunneling model which includes the possible resonant-mode excited states.

The three observed absorption lines in $\mathrm{RbCl}: \mathrm{Ag}^{+}$ as well as their electric field and stress dependence are found to be consistent with a [110] offcenter position for the $\mathrm{Ag}^{+}$ions. This disagrees with the electrocaloric results of Kapphan and Luty, where a [111] dipole orientation has been determined. The reason for this discrepancy is not yet resolved.

The complicated absorption spectrum of $\mathrm{NaCl}$ : $\mathrm{OH}^{-}$is interpreted in terms of a model which incorporates both librational tunneling states as well as resonant-mode energy states. The vibrational excited state is assumed to be split by the $C_{4 v}$ symmetry of the local potential wells. The inclusion of tunneling then gives a total of seven excited-state energy levels. The four tunneling parameters and two resonant-mode energy parameters are used to fit the model to the observed absorption spectrum, which consists of at least eight lines. With the exception of one or possibly two predicted absorption lines which are not observed, the model correctly gives the transition frequencies and temperature dependences of the observed lines. The relative absorption strengths of lines do not agree with those predicted by the model. The three measurable electric-field-induced frequency shifts can be fitted with the model calculations assuming an $\mathrm{OH}^{-}$dipole moment of $0.9 \mathrm{e \AA}$. For the electric-field-induced dichroisms, no such quantitative comparison can be made, but there is considerable qualitative agreement.

In conclusion, we see that the far-infrared properties of paraelectric impurities are, in general, more complex and difficult to understand than the more straightforward vibrational character of lines observed for on-center impurities. ${ }^{1-3}$ On the whole, the very-low-lying states resulting 


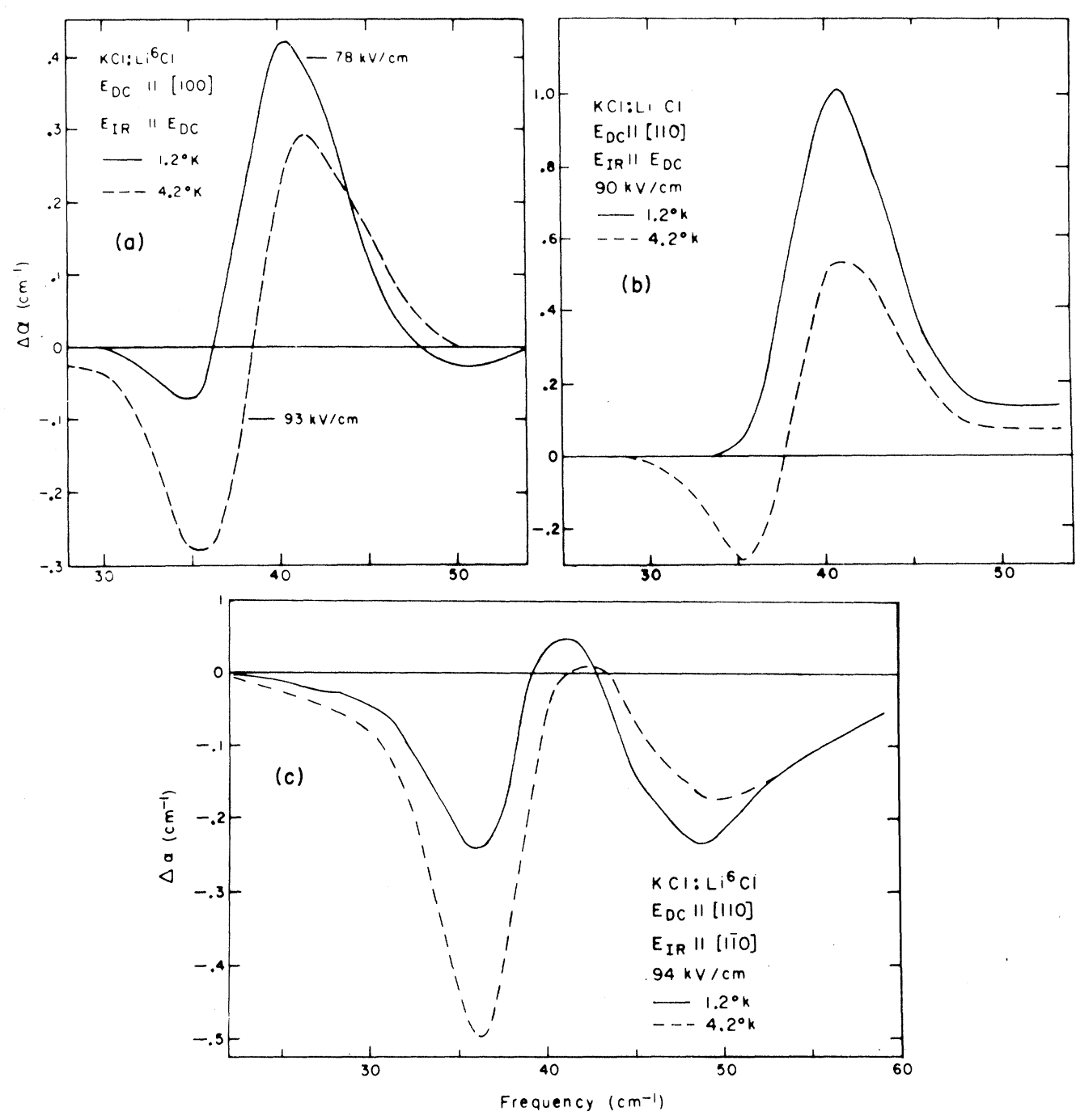

FIG. 27. (a) [100] electric field dependence of the $\mathrm{KCl}: \mathrm{Li}^{6} \mathrm{Cl} 40-\mathrm{cm}^{-1}$ band. (b) [110] electric field dependence of

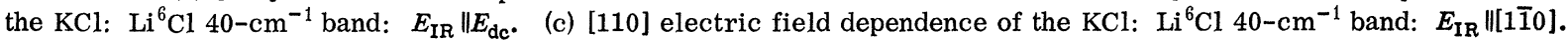

from librational tunneling between equivalent "equilibrium" orientations are well understood in terms of the tunneling model for both $\mathrm{KCl}: \mathrm{Li}$ and $\mathrm{NaCl}: \mathrm{OH}$, but no such transitions have been observed for $\mathrm{RbCl}$ : Ag. This may be because the tunneling splitting is very small in this system. For $\mathrm{KCl}: \mathrm{Li}$ in particular, the observation of what is effectively parelectric resonance in the far infrared has strikingly confirmed the off-center model in which the $\mathrm{Li}^{+}$ion is displaced in the [111] direction. Similar results in $\mathrm{NaCl}: \mathrm{OH}$ suggest that in this system the tunneling splitting is about $2 \mathrm{~cm}^{-1}$. In contrast to the tunneling transitions themselves, transitions from the tunneling multiplet to higher excited states of the systems studied are not quantitatively well understood. There is some measure of agreement with the models we have proposed for all three systems, but the detailed nature of the excited states involved and their response to external fields must remain somewhat of a mystery at this stage.

\section{APPENDIX: ELECTRIC FIELD DEPENDENCE OF $\mathrm{KCl}: \mathrm{Li}^{+}$EXCITED STATES}

Figure 27 (a) shows the electric-field-induced absorption spectrum for $\mathrm{KCl}: \mathrm{Li}^{6} \mathrm{Cl}$ with an external $[100]$ field, and $E_{\mathrm{IR}} \| E_{\mathrm{dc}}$. Measurements were made at both 1.2 and $4.2{ }^{\circ} \mathrm{K}$. The $1.2^{\circ} \mathrm{K}$ measurement shows a small decrease in absorption near $36 \mathrm{~cm}^{-1}$, and a large increase in absorption near $41 \mathrm{~cm}^{-1}$. At $4.2^{\circ} \mathrm{K}$, the $41-\mathrm{cm}^{-1}$ increase becomes smaller while the $36-\mathrm{cm}^{-1}$ decrease becomes larger. Very few conclusions can be drawn with any 
certainty from this data. The $41-\mathrm{cm}^{-1}$ absorption increases in frequency with increasing field. It cannot be attributed primarily to a shift of an existing absorption line because there is no corresponding large decrease in absorption at $1.2^{\circ} \mathrm{K}$. It is most likely an initially forbidden transition becoming allowed in the presence of the field. The temperature dependence indicates that the initial state for this transition (or these transitions) is the $A_{1 g}$ level of the ground-state tunneling multiplet.

The temperature dependence of the decrease at $36 \mathrm{~cm}^{-1}$ indicates that it arises from a transition for which the initial state is not the $A_{1 g}$ ground state.

Figure 27 (b) shows $\Delta \alpha$ for $\mathrm{KCl}: \mathrm{Li}^{6} \mathrm{Cl}$ with an external $[110]$ field, and $E_{\mathrm{IR}} \| E_{\mathrm{dc}}$. The $\mathrm{Li}^{6}$ concentration for this sample is $5 \times 10^{18} \mathrm{Li}^{+} / \mathrm{cm}^{3}$. At $1.2^{\circ} \mathrm{K}$ there is an increase in absorption near 41 $\mathrm{cm}^{-1}$, and no decrease in absorption. At $4.2^{\circ} \mathrm{K}$, the increase at $41 \mathrm{~cm}^{-1}$ becomes weaker, and a decrease in absorption near $36 \mathrm{~cm}^{-1}$ appears. These results are qualitatively similar to those for a $[100]$ field.

Figure 27 (c) shows $\Delta \alpha$ for $\mathrm{KCl}: \mathrm{Li}^{6} \mathrm{Cl}$ with $E_{\mathrm{dc}} \|\lfloor 110]$ and $E_{\mathrm{IR}} \|[1 \overline{1} 0]$. In this case, there is no increase in absorption at $41 \mathrm{~cm}^{-1}$. The decrease at $36 \mathrm{~cm}^{-1}$ is very strong, and in addition, there is a decrease in absorption near $49 \mathrm{~cm}^{-1}$.

At this point, very little can be said about these results, except that they are qualitatively the kind of results we would expect for a tunneling system. It is clear that if we are to interpret these electric field dependences we must better understand the $\mathrm{KCl}: \mathrm{Li}^{+}$excited-state energy levels.

\footnotetext{
*Work supported by U.S. Atomic Energy Commission under Contract No. AT(30-1)-2391, Technical Report No. NYO-2391-109. Additional support was received from the Advanced Research Projects Agency through the Materials Science Center at Cornell University, Report No. 1314.

$\dagger$ Present address: Department of Physics, University of Illinois, Urbana, Ill.

+Harkness Fellow of the Commonwealth Fund of New York, on leave from the Atomic Energy Research Establishment, Harwell, Didcot, Berkshire, U.K.

§Present address: Atomic Energy Research Establishment, Harwell, Didcot, Berkshire, U. K.

${ }^{1}$ R. D. Kirby, I. G. Nolt, R. W. Alexander, Jr., and A. J. Sievers, Phys. Rev. 168, 1057 (1968).

${ }^{2}$ I. G. Nolt and A. J. Sievers, Phys. Rev. 174, 1004 (1968).

${ }^{3}$ R. W. Alexander, A. E. Hughes, and A. J. Sievers, Phys. Rev. B 1, 1563 (1970).

${ }^{4}$ H. B. Shore, Phys. Rev. 151, 570 (1966).

${ }^{5}$ P. Sauer, O. Schirmer, and J. Schneider, Phys. Status Solidi 16, 79 (1966).

${ }^{6}$ M. Gomez, S. P. Bowen, and J. A. Krumhansl, Phys. Rev. 153, 1009 (1967).

${ }^{7}$ H. S. Sack and M. C. Moriarty, Solid State Commun. 3, 93 (1965).

${ }^{8}$ W. Kanzig, H. R. Hart, and S. Roberts, Phys. Rev. Letters 13, 543 (1964).

${ }^{9}$ U. Kuhn and F. Luty, Solid State Commun. 4, 31 (1965).

${ }^{10}$ G. Lombardo and R. O. Pohl, Phys. Rev. Letters 15 , 291 (1965); R. O. Pohl, V. L. Taylor, and W. M. Goubau, Phys. Rev. 178, 1431 (1969).

${ }^{11}$ I. Shepherd and G. Feher, Phys. Rev. Letters $\underline{15}$, 194 (1965).

${ }^{12}$ Kapphan and F. Luty, Solid State Commun. 6 , 907 (1968).

${ }^{13}$ W. E. Bron and R. W. Dreyfus, Phys. Rev. $\underline{163}, 304$ (1967).

${ }^{14}$ G. Feher, I. Shepherd, and H. B. Shore, Phys. Rev. Letters $\underline{16}, 500$ (1966).
}

${ }^{15}$ T. L. Estle, Phys. Rev. 176, 1056 (1968).

${ }^{16}$ R. A. Herendeen, Bull. Am. Phys. Soc. 13, 660

(1968); R. A. Herendeen and R. H. Silsbee, Phys. Rev. 188,645 (1969).

${ }^{17} \mathrm{D}$. W. Alderman and R. M. Cotts, Phys. Rev. B (to be published).

${ }^{18}$ F. C. Baumann, Bull. Am. Phys. Soc. 9, 644 (1964);

F. C. Baumann, J. P. Harrison, R. O. Pohl, and W. D. Seward, Phys. Rev. 159, 691 (1967).

${ }^{19}$ D. Walton, Phys. Rev. Letters 19, 305 (1967).

${ }^{20}$ P. P. Peressini, J. P. Harrison, and R. O. Pohl, Phys. Rev. 180, 926 (1969).

${ }^{21} \mathrm{~W} . \overline{\mathrm{D}}$. Seward and V. Narayanamurti, Phys. Rev. 148, 463 (1966); V. Narayanamurti, W. D. Seward, and R. O. Pohl, ibid. 148, 482 (1966).(1966).

${ }^{22}$ N. E. Byer and H. S. Sack, J. Phys. Chem. Solids 29 , 677 (1968).

${ }^{23}$ J. P. Harrison, P. P. Peressini, and R. O. Pohl, Phys. Rev. 171, 1037 (1968).

${ }^{24}$ A. J. Sievers, in Elementary Excitations in Solids, edited by A. A. Maradudin and G. F. Nardelli (Plenum, New York, 1969), p. 193.

${ }^{25}$ I. G. Nolt, Ph. D. thesis, Cornell University, Materials Science Center Report No. 765, 1967 (unpublished).

${ }^{26}$ K. F. Renk, Phys. Letters 20, 137 (1966).

${ }^{27}$ D. R. Bosomworth, Solid State Commun. $\underline{5}, 681$ (1967).

${ }^{28}$ R. D. Kirby, A. E. Hughes, and A. J. Sievers, Phys. Letters 28A, 170 (1968).

${ }^{29}$ M. V. Klein, B. Wedding, and M. A. Levine, Phys. Rev. 180, 902 (1969).

${ }^{30} \mathrm{~B}$. Wedding and M. V. Klein, Phys. Rev. 177, 1274 (1969).

${ }^{31}$ I. G. Nolt, R. D. Kirby, C. D. Lytle, and A. J. Sievers, Appl. Opt. $\underline{8}, 309$ (1969).

${ }^{32}$ H. D. Drew and A. J. Sievers, Appl. Opt. $\underline{8}, 2067$ (1969).

${ }^{33}$ Most of the alkali-halide crystals studied were grown in the crystal growing facility of the Materials Science Center at Cornell University.

${ }^{34}$ B. P. Clayman, Ph. D. thesis, Cornell University, 
Materials Science Center Report No. 1029, 1969 (unpublished).

${ }^{35} \mathrm{G}$. V. Chester (private communication).

${ }^{36}$ A. Lakatos and H. S. Sack, Solid State Commun. $\underline{4}$, 315 (1966).

${ }^{37}$ P. Clayman, I. G. Nolt, and A. J. Sievers, Solid State Commun. 7,7 (1969).

${ }^{38}$ H. F. Macdonald, M. V. Klein, and T. P. Martin, Phys. Rev. 177, 1292 (1969).

${ }^{39}$ W. D. Wilson, R. D. Hatcher, G. J. Dienes, and R. Smoluchowski, Phys. Rev. 161, 888 (1967).

${ }^{40}$ A. F. Devonshire, Proc. Roy. Soc. (London) A153, 601 (1936).

${ }^{41}$ E. B. Wilson, Jr., J. C. Decius, and P. C. Cross, Molecular Vibrations (McGraw-Hill, New York, 1955), Chap. 7.

${ }^{42}$ T. L. Estle, B. Dischler, and R. W. Timme, Bull. Am. Phys. Soc. 14, 346 (1969).

${ }^{43} \mathrm{M}$. Gomez, Ph.D. thesis, Cornell University, Materials Science Center Report No. 910, 1968 (unpublished).

${ }^{44} \mathrm{G}$. Benedek (unpublished).

${ }^{45}$ W. Dreybrodt and K. Fussgaenger, Phys. Status Solidi $\underline{18,} 133(1966)$.
${ }^{46} \mathrm{Dr}$. B. Wedding and Professor M. V. Klein kindly gave us several $\mathrm{OH}^{-}$- and $\mathrm{OD}^{-}$-doped $\mathrm{NaCl}$ crystals which had been analyzed for $\mathrm{OH}^{-}$concentration.

${ }^{47}$ R. Rosenbaum and M. V. Klein, Phys. Rev. (to be published).

${ }^{48}$ W. R. Fenner, Ph. D. thesis, University of Illinois, 1969 (unpublished).

${ }^{49} \mathrm{Dr}$. D. R. Bosomworth supplied the $\mathrm{NaCl}$ sample doped with 1500-ppm $\mathrm{OH}^{-}$.

${ }^{50}$ Harshaw Chemical Company, Cleveland, Ohio.

${ }^{51}$ Dr. R. K. Skogerboe and the Materials Science Center Analytical Facility analyzed the samples for impurity concentration using flame-emission techniques.

${ }^{52}$ P. Sauer, Z. Physik 194, 360 (1966).

${ }^{53}$ C. K. Chau, M. V. Klein, and B. Wedding, Phys. Rev. Letters 17, 521 (1966).

${ }^{54}$ H. B. Shore, Phys. Rev. Letters 17, 1142 (1966).

${ }^{55}$ M. E. Baur and W. R. Salzman, Phys. Rev. $\underline{151}, 710$ (1966).

${ }^{56}$ R. S. Scott and W. H. Flygare, Phys. Rev. $\underline{182}, 445$ (1969).

${ }^{57}$ A. M. Stoneham, Proc. Roy. Soc. (London) 89, 909 (1966).

\title{
Formation of $F_{2}^{+}$Centers in $\mathrm{KI}^{\dagger}$
}

\author{
Alexander Kouvalis \\ Zenith Radio Corporation, Chicago, Mlinois 60639 \\ (Received 26 January 1970)
}

\begin{abstract}
When undoped $\mathrm{KI}$ is $\mathrm{x}$ rayed at $130^{\circ} \mathrm{K}$ and then irradiated with $F$ light at 240 or $270^{\circ} \mathrm{K}$, the $M$ and $R$ centers are formed. Subsequent irradiation at $78^{\circ} \mathrm{K}$ with $M_{L}$ light results in the formation of an optical-absorption band at $1650 \mathrm{~nm}$ at the expense of the $M$ band. Studies suggest that it is associated with $F_{2}^{+}$centers (formerly called $M^{+}$), which are formed at $78^{\circ} \mathrm{K}$ by (i) $V+F_{2}+\left(M_{L}\right.$ light $) \rightarrow V^{-}+F_{2}^{+}$(where $M_{L}$ light represents light absorbed by the $M_{L}$ bands) and destroyed by $\mathrm{x}$ rays at $78^{\circ} \mathrm{K}$, by (ii) $V^{-}+F_{2}^{+}+$(x rays) $\rightarrow V+F_{2}$ [where $V^{-}$is an electron center containing a halogen $(V)$ center core]. $F_{2}^{+}$centers may also be destroyed at $78^{\circ} \mathrm{K}$ by $F$ light, by (iii) $V^{-}+F_{2}^{+}+F+h \nu_{F} \rightarrow V^{-}+F_{2}+\alpha$. Studies show that the $F_{2}^{+}$center is not formed optically as a result of $V_{K}$ excitation, but only by direct ionization. The formation of $F_{2}^{+}$centers by $M_{L}$-light irradiation in $x$-rayed colored crystals seems to depend on the existence of $V$ centers which act as special electron traps.
\end{abstract}

\section{INTRODUCTION}

It is well known that $F$-band irradiation of alkali halides near room temperature results in the formation of $M, R$, and $N$ bands. The $M, R$, and $N$ bands are called $F$-aggregate centers and are formed on the longer wavelength side of the $F$ band. ${ }^{1}$ The various $F$-aggregate centers so far mentioned are electrically neutral clusters of anion vacancies and trapped electrons. If additional electrons are trapped (by $F$-aggregate centers), one would have $F_{2}^{\prime}, F_{3}^{\prime}$, and $F_{4}^{\prime}$ centers (where the prime denotes an additional electron) having an effective negative charge. ${ }^{2}$ On the other hand, if electrons are removed from $F$-aggregate centers, one would have $F_{2}^{+}, F_{3}^{+}$, and $F_{4}^{+}$centers having effective positive charge. With either positively or negatively charged $F$-aggregate centers, one must have complementary centers of opposite charge in the crystal. Recently, a number of studies have been reported on absorption bands associated with ionized $F$-aggregate centers $\left(F_{2}^{+}\right.$and $\left.F_{3}^{+}\right)$in several alkali halides. ${ }^{3-8}$

The present work deals specifically with the observations of $\mathrm{F}_{2}^{+}$centers in $\mathrm{KI}$ and with the mechanism of formation and destruction of $\mathrm{F}_{2}^{+}$centers. 